\title{
1 Optimization of lag phase shapes the evolution of a bacterial enzyme
}

2

3 Bharat V. Adkar ${ }^{1}$, Michael Manhart ${ }^{1}$, Sanchari Bhattacharyya ${ }^{1}$, Jian Tian ${ }^{1,2}$, Michael

4 Musharbash $^{1}$, Eugene I. Shakhnovich ${ }^{1 *}$

$5 \quad{ }^{1}$ Department of Chemistry and Chemical Biology, Harvard University, 12 Oxford St., 6 Cambridge, MA 02138, USA

$7 \quad{ }^{2}$ Biotechnology Research Institute, Chinese Academy of Agricultural Sciences, 12

8 Zhongguancun South Street, Beijing, 100081, China

$9 \quad{ }^{*}$ Correspondence should be addressed to E.S. (shakhnovich@chemistry.harvard.edu) 


\section{Abstract}

12 Mutations provide the variation that drives evolution, yet their effects on fitness remain 13 poorly understood. Here we explore how mutations in the essential enzyme Adenylate 14 Kinase (Adk) of $E$. coli affect multiple phases of population growth. We introduce a 15 biophysical fitness landscape for these phases, showing how they depend on molecular and 16 cellular properties of Adk. We find that Adk catalytic capacity in the cell (product of 17 activity and abundance) is the major determinant of mutational fitness effects. We show 18 that bacterial lag times are at a well-defined optimum with respect to Adk's catalytic 19 capacity, while exponential growth rates are only weakly affected by variation in Adk. 20 Direct pairwise competitions between strains show how environmental conditions modulate 21 the outcome of a competition where growth rates and lag times have a tradeoff, altogether

22 shedding light on the multidimensional nature of fitness and its importance in the 23 evolutionary optimization of enzymes. 


\section{Introduction}

Random mutagenesis is often used to assess the distribution of fitness effects in simple experimental models such as propagating viruses and microbes evolving under antibiotic stress $^{1,2}$. However, the enormous size of sequence space severely constrains how much of the fitness landscape can be explored this way, and mechanistic and predictive insights from these experiments are further limited by a lack of knowledge of the molecular effects of mutations. Instead, a more targeted experimental approach relies on the concept of a biophysical fitness landscape, in which fitness effects of mutations are mapped through their effects on molecular traits of the mutated proteins. In this approach, biophysically-rational genetic variation is introduced on the chromosome, and the molecular and phenotypic effects of that variation are analyzed concurrently ${ }^{3-6}$. By mapping fitness effects to variation of molecular properties rather than to sequences of mutated proteins, we can dramatically reduce the dimensionality of the genotype-to-phenotype mapping. The underlying hypothesis is that variation in a small number of properly-selected molecular traits of mutated proteins can explain most of the resulting mutational variation in fitness, and that the relationship between these molecular traits and fitness is smooth and continuous. Several recent studies have supported this approach ${ }^{5-7}$.

The relationship between sequence variation and fitness is further confounded by the fact that multiple life-history traits contribute to fitness ${ }^{8}$, and the relative importance of these traits to the long-term evolutionary fate of a mutation may be highly dependent on environmental and ecological conditions. While multicellular organisms are generally described by a large number of traits (e.g., viability at various life phases, mating success, fecundity, etc.), unicellular microorganisms like bacteria and yeast are described by relatively fewer components of fitness, such as the time in lag phase, the exponential growth rate, and the overall yield at saturation. All these phases of growth contribute toward the outcome when in competition for limited resources, and hence determine fitness ${ }^{3,9}$. The relative importance of these different phases of bacterial growth in sculpting the fitness landscape depends on the conditions of growth and competition ${ }^{10-}$ 12 .

2 Overall, the challenge in quantitatively characterizing the fitness landscape is twofold: 3 Understanding fitness in terms of contributions from different phases of growth, and linking each of these components to molecular and cellular traits. In this work, we address both challenges by 
introducing biophysically-rational genetic variation in the $a d k$ locus that encodes the essential E. coli enzyme Adenylate Kinase (Adk), and projecting the ensuing variations of fitness effects (phenotypic components like growth rate and lag time) onto the biophysical traits of Adk. We find that a unique combination of molecular and cellular traits of Adk - the product of intracellular abundance and catalytic activity, which we term catalytic capacity - provides a reliable predictor of fitness effects across the full range of phenotypic variation. Furthermore, we find that the length of the lag phase is more sensitive to variation in Adk catalytic capacity than is the exponential growth rate, so that the lag phase of the wild-type E. coli appears to be optimal with respect to variation in Adk catalytic capacity.

\section{Results}

\section{Biophysical properties of Adk mutants}

Destabilizing mutations have been shown to cause a drop in intracellular protein abundance, mostly through a decrease in the folded fraction of the protein ${ }^{3}$. Hence in order to sample a broad range of molecular and cellular traits of Adk protein below the wild-type levels, we chose a set of 21 missense mutations at 6 different positions of adk. (Table S1 and Fig. 1). We selected residues such that their accessible surface area was less than $10 \%$ and they were at least $6 \AA$ away from the catalytically-active sites of Adk, so that mutations at these residues were likely to destabilize the protein ${ }^{13}$. For most mutants, we chose amino acid mutations that appeared only at low frequency in an alignment of 895 homologous sequences of Adk. As intended, the purified mutant proteins were destabilized over a wide range $\left(\sim 17^{\circ} \mathrm{C}\right.$ in terms of $\mathrm{T}_{\mathrm{m}}$, and $\sim 7.5 \mathrm{kcal} / \mathrm{mol}$ in terms of folding $\Delta G$ ) (Table S1, Figs. 1B, S1, S2). In only one case (L209I) did we change the E. coli sequence to the consensus amino acid at that position, and we found it in fact stabilized the protein by $\sim 1 \mathrm{kcal} / \mathrm{mol}$ (Table S1). Although most of the Adk mutants were less stable than the wild-type (WT), they nevertheless existed predominantly as monomers in solution (Fig. S3). However, several mutations in one position - V106H, V106N, and V106W - did have significant fractions of proteins present in higher oligomeric forms, in addition to the predominant monomeric species (Fig. S3). These proteins bound 4,4'-Dianilino-1,1'-binaphthyl5,5'-Disulfonate (Bis-ANS) dye to a higher degree compared to the rest of the mutants (Fig. S4), indicating the presence of possible molten globule states in solution ${ }^{14}$. The proteostat dye that reports on protein aggregation ${ }^{4,15}$ also bound these mutants more strongly compared to others 
85 (Fig. S4), clearly indicating a higher fraction of aggregated species. The catalytic efficiency

$86\left(k_{c a t} / K_{M}\right)$ of the mutant Adk proteins was distributed broadly with most mutants showing a

87 lower activity than E. coli WT (Table S1, Figs. 1C, S5).

\section{Intracellular abundance of Adk follows prediction from Boltzmann distribution}

We then incorporated each of the 21 adk mutations one-by-one into the E. coli chromosome using a genome-editing approach based on homologous recombination ${ }^{3,4}$. We measured the total intracellular abundance of WT and mutant Adk proteins using a quantitative western blot (Table S2). The sigmoidal dependence of total intracellular Adk abundance on folding stability $(\Delta G)$ (Fig. 1D) is well-described by the Boltzmann distribution for two-state unfolding proteins:

$$
P_{F}=\frac{1}{1+\exp (\beta \Delta G)}
$$

where $P_{F}$ is the fraction of folded molecules in the ensemble of intracellular Adk and $b=1 / k_{B} T$, with Boltzmann constant $k_{B}$ and growth temperature $T$. The total measured abundance of a protein is its amount in the cytoplasm at steady-state, achieved by a balance between production and degradation. Since Adk is expressed from a constitutive promoter in the cells, it is generally safe to assume that the rates of production of all mutants are similar. Under this assumption, the sigmoidal dependence of abundance on stability clearly indicates that the unfolded protein is degraded in the active medium of the cytoplasm.

\section{Mutations in Adk affect lag times more than exponential growth rates}

Mutations in Adk affect both intracellular abundance (via folding stability) and catalytic activity of the protein. Flux dynamics theory predicts, and experiments have confirmed, that the key enzymatic parameter determining the flux through an enzymatic reaction chain is the quantity which we call "catalytic capacity," defined as the product of intracellular abundance and enzymatic efficiency $k_{c a t} / K_{M} 5,6,16$. To that end, we determined how two key components of bacterial growth - the exponential growth rate and the lag time (Fig. 2A) - depend on the total catalytic capacity of Adk in E. coli cells (Fig. 2B,C; also see Methods and Fig. S6-S8 for estimation of growth parameters). We find that while only 3 out of 21 strains show a drop in 
growth rate greater than 5\% of WT, 17 strains show an increase in lag time for a similar change over the WT value (Table S2). This suggests that the mutations in Adk affect the lag phase more significantly than the exponential growth phase. One mechanism for producing longer apparent lag times is when a greater proportion of cells that come out of stationary phase are simply nonviable, as described in a recent study ${ }^{17}$. However, this appears not to be the major cause in our case, as lag times are fairly consistent across replicates (error bars in Fig. 2C) and do not negatively correlate with the number of viable cells (Fig. S9). We also find that the variation in total catalytic capacity of Adk correlates better with the variation in lag times (Spearman's rank correlation $\rho=-0.44, p=0.057$ ) than with the variation in growth rates (Spearman's rank correlation $\rho=-0.08, p=0.737$ ) (Fig. S10). The variation in lag times is also better explained by the variation in catalytic capacity than with any of the Adk properties separately (stability, abundance, or activity) (Fig. S10). Surprisingly, growth rate appears to tolerate a rather large drop in catalytic capacity of Adk, while lag time does not.

\section{WT E. coli is positioned at the cusp of the biophysical fitness landscape for lag time}

Since almost all the designed mutants were destabilizing and therefore have lower catalytic capacity than E. coli WT, they only provide sampling in the lower range of catalytic capacity. In studies so far, no evidence exists for changes in intracellular protein abundance for stabilizing mutations. Hence to determine the dependence of growth rate and lag time on catalytic capacity above WT levels, we over-expressed WT Adk from a pBAD plasmid (see Supplementary Methods). We observed no significant change in either growth rate or lag time at higher than endogenous catalytic capacity (Fig. 2B,C, S8, and Table S3). This means that while the growth rate appears to be insensitive to large changes in Adk catalytic capacity both below and above the wild-type level, WT catalytic capacity appears to be situated at the threshold of optimizing lag time. Next, we attempted to quantitatively compare the position of WT on these two fitness landscapes. To that end, we used a simple reciprocal Michaelis-Menten-like function to fit the relative growth times (growth time is reciprocal of growth rate ${ }^{\mu}$ ) and lag times (Fig. S11, also see Eq. 3 and Methods). The fitting parameter $b$ which characterizes the onset of curvature on the landscape (analogous to $K_{M}$ in Michaelis-Menten equation for enzymatic rate) reports proximity of WT to the cusp on the landscape (see Methods). It was 0.006 for growth time and 
0.019 for lag time as compared to normalized catalytic capacity of 1 for WT. This shows that WT is situated closer to the cusp in terms of lag time as compared to growth time or growth rate.

\section{Shorter lag imparts advantage at low carrying capacity: A computational model}

This data highlights the pleiotropic effects of mutations on different phases of bacterial population growth, which raises the question of how pleiotropy shapes the evolutionary fate of a mutation. We explore this issue by considering the outcome of binary competitions between strains ${ }^{18}$. We first simulated binary competitions over a wide range of growth rates and lag times in media conditions that allow for either 5-fold (low carrying capacity) or 500-fold (high carrying capacity) increase over the initial population (Fig. 3A) (See Methods). We found that there is a significant tradeoff between lag times and growth rates in determining the winners of binary competitions, with lag playing a more important role at low carrying capacity (Fig. 3A), implying that beneficial lag provides a greater fitness advantage under strongly nutrient-limiting conditions.

\section{Shorter lag imparts advantage at low carrying capacity: Experimental evidence}

To realize varying nutrient conditions in binary competition experiments, we explored the growth of E. coli over a range of glucose concentrations, mimicking the variation of carrying capacity in simulations, and found that only the carrying capacities are proportional to glucose concentration with minimal effects on lag time and growth rate (Fig. 4). This suggests that observing the outcome of the competition at different time snapshots in a nutrient-rich medium is equivalent to running the competition at different glucose concentrations (carrying capacities). To evaluate the predictions from simulations, we carried out two sets of binary competition experiments based on the overall distribution of growth rates and lag times (Fig. 3B). First, we selected strains exhibiting a tradeoff between growth rate $(\mu)$ and lag time $(\lambda)\left(\mu_{1}>\mu_{2}\right.$ and $\lambda, 1>\lambda, 2$ ) (inset of Fig. 5B). Second, we tested competition between strains that differ in their lag times but have nearly indistinguishable growth rates $\left(\mu_{1} \approx \mu_{2}\right.$ and $\left.\lambda_{1}>\lambda_{2}\right)$ (inset of Fig. 5C). In all cases a strain with shorter lag time is expected to dominate at lower carrying capacity conditions (corresponding to the competition outcome at early time points), however this advantage would be lost at later time points if its growth rate is lower than that of the competing strain (Fig. 5A). In the second scenario, the advantage due to short lag is expected to persist even 
at high carrying capacity conditions because the growth rates of the competing strains do not differ. We estimated the relative proportions of the two strains by a qPCR-based mismatch amplification mutation assay (MAMA) $\operatorname{approach}^{19}$ (see Methods and Fig. S12). As expected in the first scenario, $\mathrm{L} 083 \mathrm{~F}$ and $\mathrm{V} 106 \mathrm{H}$ dominated at earlier time points when competed against A093I and L209I, respectively, due to their shorter lag times $\left(\lambda_{\mathrm{L} 083 \mathrm{~F}}<\lambda_{\mathrm{A} 093 \mathrm{I}}\right.$ and $\left.\lambda_{\mathrm{V} 106 \mathrm{H}}<\lambda_{\mathrm{L} 209 \mathrm{I}}\right)$ (Fig. 5B). Eventually their fraction dropped below 0.5 at later time points (equivalent to high carrying capacity) where the growth rates determine the competition output $\left(\mu_{\mathrm{L} 083 \mathrm{~F}}<\mu_{\mathrm{A} 093 \mathrm{I}}\right.$ and $\mu \mathrm{V} 106 \mathrm{H}<\mu \mathrm{L} 209 \mathrm{I})$ (Fig. 5B). Similarly, for the second scenario, despite having similar growth rates ( $\mu_{\mathrm{WT}} \approx \mu_{\mathrm{Y} 182 \mathrm{~V}} \approx \mu_{\mathrm{L} 209 \mathrm{~A}}$ ), the fraction of WT was always maintained above 0.5 as it spends a shorter time in the lag phase compared to Y182V and L209A (Fig. 5C). The early advantage to WT due to its shorter lag phase determined the competition fitness throughout the whole growth cycle.

\section{Discussion}

A complete mapping of mutational fitness effects would require sampling a practically infinite number of mutations, an impossible proposition. Instead, we can project fitness onto a fairly small number of molecular properties of proteins ${ }^{5-7,20}$. Within this paradigm, the identity of a particular mutation does not matter as much as its effect on essential biochemical and biophysical properties of the proteins in question. Our 21 engineered mutations in Adk, along with the overexpression data, allow us to outline the biophysical fitness landscape, covering a wide range of variation of the physical parameters of the Adk protein. This data shows that we can collapse several molecular phenotypes into a single effective parameter - the product of protein abundance and activity $k_{c a t} / K_{M}$ (catalytic capacity) - which quantitatively determines the biophysical fitness landscape to a great extent (Fig. 2B,C). That is, Fig. 2 indicates that the fitness effects of mutations can largely be predicted from their biophysical effects over a broad range of catalytic capacity. Indeed, Adk catalytic capacity explains the variation in lag times to a large extent (Fig. S10), validating the utility of a biophysical fitness landscape for mapping fitness effects.

These results illustrate how the evolutionary endpoint of molecular traits may depend fundamentally on the multidimensional nature of fitness, with the relative importance of different 
components of fitness depending on the environment and lifestyle of the organism. It has been argued that endogenous molecular traits are established as a result of mutation-selection balance $^{21}$, with the final outcome depending on the relative strengths of selection and genetic drift as determined by the population structure ${ }^{22,23}$. Here we encounter a more complex situation where mutations in the essential enzyme Adk change multiple fitness components. In this case, the mutation-selection balance apparently resulted in disparate outcomes for the two fitness components with respect to the molecular trait, placing lag time at the cusp while keeping the exponential growth rate farther within the plateau region of its respective biophysical fitness landscape. Such an outcome may reflect different strengths of selection on growth and lag. The relative strength of selection on these fitness components depends crucially on the environmental conditions (e.g. nutrient availability, etc.) ${ }^{24}$. Our studies of binary competitions (Figs. 3 and 5) highlight this scenario by showing how the environmental parameter of carrying capacity can determine winners and losers in evolutionary dynamics. Although the lag time of a population can depend not only on the environment but also on the population's specific history (e.g., how long it was previously in stationary phase), the fundamental role of Adk in metabolism suggests that its effects on lag time are likely to be common across conditions and histories. The deep connection between ecological history of species and optimization of biophysical traits of their proteins is a subject for valuable future studies.

Much of our current understanding of microbial cultures and fitness comes from experiments done in the laboratory, where strains are typically grown under a large supply of nutrients. The situation might be very different in the wild, however, where bacteria and other microbes have to survive under harsh conditions of nutrient starvation, extreme temperature, and other environmental stresses ${ }^{25-27}$. For example, E. coli is the predominant facultative anaerobe in the gastrointestinal tract ${ }^{28}$ which allows it to thrive in fluctuating environments of differing oxygen concentrations along the GI tract (e.g., the small vs. the large intestine). In these circumstances, organisms are likely to spend only a minute fraction of their life-cycle in the exponential growth phase, while undergoing many cycles of lag-growth-saturation as new resources become available and old ones are exhausted. It is therefore intuitive to expect that there has been strong selection in favor of organisms that can not only divide rapidly during exponential growth, but that can also wake up quickly from their lag phase and respond to newly available resources. Our study demonstrates how this selection may shape individual molecular traits. 
229 This work highlights the relationship between various components of fitness and the molecular

230 properties of modern enzymes - the endpoint of evolutionary selection. An interesting question

231 which is beyond the scope of current work is how modern variants emerged in evolutionary

232 dynamics. To that end mapping temporary reconstructed ancestral species onto biophysical

233 fitness landscape of Adk (and other enzymes) appears a promising direction of future research.

\section{$235 \underline{\underline{\text { Methods }}}$}

236 Selection of mutations: Mutations at relatively-buried positions generally result in decreased 237 stability and lower fitness ${ }^{13,29}$. Hence we selected the sites for mutagenesis with side-chain 238 accessibility of less than 10\%. In addition, the selected sites were also away from the active-site 239 residues, or active-site contacting residues, and a minimum of $6 \AA$ away from the inhibitor Ap5A 240 binding sites (pdb lake). The structure of Adk is divided into three domains: LID (residues 118241 160), NMP (residues 30-67), and Core (residues 1-29, 68-117, and 161-214). We define the 242 active-site residues as those whose accessible surface area changes by at least $5 \AA^{2}$ in the 243 presence of the inhibitor Ap5A. A similar criterion was used to define the residues contacting the 244 active site. Altogether 4 residues from the LID domain, 3 from the NMP domain, and 28 from 245 the Core domain satisfy these criteria. Of the 28 sites from the Core domain, we randomly chose 2466 to mutate. We chose the identities of the mutations to span various sizes of the side chains and 247 a range of conservation. We derived the conservation from the multiple sequence alignment of 248895 sequences for Adk collated from ExPASy database (as of Nov 2012).

249 Generation of mutant strains: We generated the strains with WT and mutant adk with 250 chloramphenicol- and kanamycin-resistance genes on either end of the adk gene using the 251 genome editing approach as described previously ${ }^{3}$. Since the $a d k$ gene is flanked by two repeat 252 regions (REPt44 and REPt45) on the wild-type chromosome, we extended the homology 253 required for recombination up to the middle of the adjacent genes.

254 Growth curve measurements and media conditions: WT and mutant strains were grown 255 overnight at $30{ }^{\circ} \mathrm{C}$ from single colonies in a supplemented M9 medium $(0.2 \%$ glucose, $1 \mathrm{mM}$ $256 \mathrm{MgSO}_{4}, 0.1 \%$ casamino acids, and $0.5 \mu \mathrm{g} / \mathrm{ml}$ thiamine). OD600 was measured for all the strains 257 and then the cultures were normalized to whichever had the lowest OD. The normalized cultures 
272 273 plots:

$$
\ln (O D)=\ln \left(O D_{0}\right)+K \exp \left[-\exp \left(-\frac{t-\lambda}{b}\right)\right]
$$

275 where the carrying capacity is $K$, the maximum growth rate is $\mu=K /(b \cdot \exp (1))$, and the lag 276 time $\lambda$ is the time taken to achieve the maximum growth rate.

277 For both the methods, we considered only data points with $O D 600 \geq 0.02$. The instantaneous 278 derivatives of all growth curves show presence of a distinct peak at OD600 values greater than 2790.02 (Fig. S6), indicating monoauxic growth and also asserting that the derived growth 280 parameters are unaffected due to ignoring the lower OD data. 
281 The $\mu$ and $\lambda$ estimated from the two aforementioned methods are strongly correlated 282 (Pearson's $r=0.80, p=1.4 \mathrm{e}-5$ for $\mu$, and $r=0.71, p=3.0 \mathrm{e}-4$ for $\lambda$ ) (Fig. S7). However, the 283 uncertainty in the fitted parameters appears to be less than the uncertainty in the parameters 284 obtained from the derivatives, which are limited by the low time-resolution of the experimental 285 data (acquired at an interval of $15 \mathrm{~min}$ ).

286 The growth rate $(\mu)$ and lag time $(\lambda)$ appear to be statistically independent of each other across 287 the Adk mutant strains (Spearman's $\rho=0.31, p=0.15$, Fig. 3B). Hence it is conceivable that 288 selection can act separately on these two traits, which is further illustrated by the different fitness 289 landscapes observed when projected onto the axis of catalytic capacity (Fig. 2B,C).

290 Statistical tests for mutational variation in growth and lag phases: We estimated the monotonic 291 relationship between various growth traits and molecular/cellular properties of Adk mutant 292 proteins using Spearman's rank correlation (Fig. S10). The agreement between growth 293 parameters derived using instantaneous derivatives and Gompertz fit were estimated by 294 Pearson's correlation coefficient (Fig. S7). We excluded V106N from all statistical analysis and 295 data fitting as its lag time is $\sim 13$ s.d. away from the average lag time of all other strains.

296 Quantification of the location of WT on the fitness landscapes: A Michaelis-Menten-like 297 elasticity curve function has been used previously, $5,6,16,20$ to fit the dependence of growth rate on 298 catalytic capacity. Since we are considering growth and lag times rather than rates, we use a 299 reciprocal form of the Michaelis-Menten-like function for fitting relative growth time $\left(\tau / \tau_{W T}\right)$ 300 and relative lag time $\left(\lambda / \lambda_{W T}\right)$ vs. catalytic capacity (Fig. S11):

$$
\text { Relative growth trait }=\frac{a \cdot(b+\text { Catalytic Capacity })}{\text { Catalytic Capacity }}
$$

302 where $a$ is the asymptotic value of the trait for infinitely large catalytic capacity, and $b$ is the 303 catalytic capacity when the trait equals twice the asymptotic value $(2 a)$. Since catalytic capacity 304 is normalized by WT, $b$ serves as a measure of how close to the cusp the WT on the respective 305 landscapes is. For fits in Fig. S11, we empirically set $a=1$ which enables easy comparison of 306 parameter $b$ for lag time and growth time plots. 
307 Simulation of binary competition: We simulated the competition of two strains by using the 308 Gompertz function (Eq. 2) to model the growth of individual strains. The initial population 309 (OD0) for both strains was equal, and growth ceases when $\Sigma\left(O D_{t} / O D_{0}\right)_{i}=K$, where $K$ is the 310 carrying capacity. We considered two different values of carrying capacities (5 and 500). We set $311 \mu_{1}$ and $\lambda_{1}$ to values derived experimentally for WT Adk strain (Table S2), while the growth 312 rates and lag times for the second competing strain were varied randomly across the intervals 3130.005 to $0.030 \mathrm{~min}^{-1}$ (for growth rate) and 50 to $300 \mathrm{~min}$ (for lag time).

314 Binary growth competition and quantification: The overnight cultures for individual strains were 315 grown for 16 hours at $30^{\circ} \mathrm{C}$. These cultures were mixed in 1:1 proportion, diluted to an OD of 3160.01 in fresh supplemented M9 media, and then regrown at $37{ }^{\circ} \mathrm{C}$. The samples were drawn at 317 different time points, and the OD was adjusted to 2.0, either by concentration or dilution. $5 \mu 1$ of 318 OD 2.0 culture was eventually diluted in $45 \mu 1$ of lysis solution (QuickExtract DNA extraction 319 solution (Epicentre)) to reach OD 0.2. Genomic DNA extracted from $50 \mu 1$ of OD 0.2 culture 320 321 was diluted 5000 times and used as template. The individual strains in the competition were differentially amplified using allele-specific primers and quantified by a qPCR-based mismatch amplification mutation assay method ${ }^{19}$ using QuantiTect SYBR Green PCR kit (Qiagen). A 150 bp long non-mutagenic amplicon of $a d k$ gene was amplified as a reference to quantify total genomic DNA. The fraction of the competing strains was determined using the following equation:

$$
\text { fraction }=2^{\wedge}\left(\left(C_{t, r e f}-C_{t, 1}\right)_{\text {competition }}-\left(C_{t, r e f}-C_{t, 1}\right)_{\text {pure }}\right)
$$

327 where ${ }^{C_{t}}$ represents threshold cycle of qPCR, ref and 1 are the PCR reactions for amplifying 328 the reference and the first allele in competition, while competition and pure represent the 329 condition of culture.

330 Data availability: All raw data for growth curves of $a d k$ WT and mutant strains, as well as WT 331 overexpression in E. coli BW27783 strains, is included as Dataset 1. 


\section{Figure Legends:}

Fig. 1: Biophysical and intracellular properties. (A) Crystal structure of Adenylate Kinase from E. coli (PDB ID 4ake ${ }^{30}$ ). The core domain is colored in green, while the LID and NMP domains are shown in white. The $\mathrm{C}_{\alpha}$ atoms of active-site residues are shown in pink, and the blue spheres represent the $\mathrm{C}_{\alpha}$ atoms of the 6 buried positions which were mutated in this study. (B) Histogram showing the distribution of folding free energies for all mutant proteins, as determined by isothermal urea denaturation at $25^{\circ} \mathrm{C}$. The stability of WT is marked by a dashed line. (C) Histogram of the catalytic activity parameter $k_{c a t} / K_{M}$ for all mutants. The dashed line indicates the WT value. (D) Total intracellular abundance of mutant Adk proteins as a function of $\Delta G$ at $37^{\circ} \mathrm{C}$. The abundances are normalized by the WT value. Each data point represents the mean and error bars are standard deviation over two experiments. The dashed line represents the fit to the Boltzmann distribution function described in Eq. 1, where $k_{B}$ was $1.987 \mathrm{cal} / \mathrm{mol} / \mathrm{K}$. See related Figs. S1-S5 and Table S1.

Fig. 2: Traits of population growth. (A) Schematic of estimation of lag time and growth rate. The representative data points (solid gray circles) were plotted as $\ln (\mathrm{OD})$ vs time and was fitted to a four parameter Gompertz function (Eq. 2) (cyan line). The red line is a tangent at the inflection point of the function. The slope of the tangent is considered as the growth rate $(\mu)$ and the time required to reach the maximum growth rate or the inflection point is taken as the lag time ${ }^{(\lambda)}$ (vertical dashed line). (B) Relative growth rate $\left(\mu / \mu_{W T}\right)$ and (C) relative lag time $\left(\lambda-\lambda_{W T}\right)$ as functions of catalytic capacity which is defined as abundance $\times k_{c a t} / K_{M}$. The mutant data is shown in gray circles, whereas red circles represent the BW27783 strain with varying degrees of overexpression of WT Adk from a pBAD plasmid. Data for WT is shown in green. The data points represent mean and error bars represent standard deviation of parameters derived from growth curves of 3 colonies (biological replicates) in triplicates (9 curves). See related Figs. S6-S11 and Tables S2-S3. The solid gray arrow indicates the direction of increasing fitness.

Fig. 3: Binary growth competition. The growth of individual strains was modeled as per Gompertz equation (Eq. 2). The growth parameters for strain 1 were fixed to those obtained for 
WT Adk (dashed gray lines) while those for strain 2 were generated randomly over a wide range of growth rates (0.005 to $0.030 \mathrm{~min}^{-1}$ ) and lag times (50 to $250 \mathrm{~min}$ ). (A) Contour plot showing fraction of strain 1 (WT) at saturation when the competition is carried out under two different carrying capacities (red line indicates $K=5$ while the black line indicates $K=500$ ). The dashed lines indicate neutrality region where both strains have equal proportions at saturation. The areas below the neutrality line (filled with solid lines) represent the parameter space where strain 2 wins the competition (fraction of strain $2>0.5$ ). (B) Scatter plot of growth rate $(\mu)$ versus lag time $(\lambda)$. The data points represent the mean and error bars the standard deviation of 6 to 9 measurements (see Table S2). The growth rate and lag time appear to be statistically independent of each other across the Adk mutant strains (Spearman's $\rho=0.31, p=0.15$ ).

Fig. 4: Growth curves at various nutrient concentration. (A) Growth curves of strains with WT Adk obtained under varying glucose concentrations in supplemented M9 medium. The fitted growth curve parameters are shown as functions of glucose concentration: (B) carrying capacity of $\ln (\mathrm{OD})$ as derived from Gompertz fitting, (C) relative growth rate $\left(\mu / \mu_{0.2}\right)$, and (D) relative lag time $\left(\lambda-\lambda_{0.2}\right)$. The growth rates and lag times are estimated from analysis of growth curve derivatives and are normalized relative to the respective values at $0.2 \%$ glucose concentration.

Fig. 5: Tradeoffs between lag and exponential growth in binary competitions. (A) Fraction of the first strain as a function of time in simulated binary competitions. We modeled growth of each strain using the Gompertz 4-parameter equation (Eq. 2) with experimentally measured growth rate and lag time values. The initial OD for individual strains was assumed to be 0.006 at the start of competition, and growth was assumed to saturate at OD of 0.6. Despite having similar growth rates, the fraction of WT in WT + L209I and WT + Y182V competitions was always above 0.5 owing to the advantage it gained due to shorter lag time (scenario 2 in the text). L083F and V106H dominate at earlier time points (equivalent to low carrying capacities) due to their short lag times compared to their respective competitors. However, at longer times (high carrying capacities) the advantage due to lag is lost due to their lower growth rates. (B, C) Experimental validations of the predictions in (A) using qPCR based mismatch amplification mutation assay (MAMA). The fraction of competing strains was estimated using Eq. 4. The data 
389 points are mean and error bars represent standard deviation of two measurements. See related 390 Fig. S12. The growth rates and lag times for the competing pairs are shown in insets.

391 


\section{References}

3931 Sanjuan, R., Moya, A. \& Elena, S. F. The distribution of fitness effects caused by single-nucleotide substitutions in an RNA virus. Proceedings of the National Academy of Sciences of the United States of America 101, 8396-8401, doi:10.1073/pnas.0400146101 (2004). Bershtein, S., Segal, M., Bekerman, R., Tokuriki, N. \& Tawfik, D. S. Robustness-epistasis link shapes the fitness landscape of a randomly drifting protein. Nature 444, 929-932, doi:10.1038/nature05385 (2006).

400

401

402

3 Bershtein, S., Mu, W. \& Shakhnovich, E. I. Soluble oligomerization provides a beneficial fitness States of America 109, 4857-4862, doi:10.1073/pnas.1118157109 (2012).

403

404

405

Bershtein, S., Mu, W., Serohijos, A. W., Zhou, J. \& Shakhnovich, E. I. Protein quality control acts on folding intermediates to shape the effects of mutations on organismal fitness. Mol Cell 49, 133-144, doi:10.1016/j.molcel.2012.11.004 (2013).

5 Bershtein, S. et al. Protein Homeostasis Imposes a Barrier on Functional Integration of Horizontally Transferred Genes in Bacteria. PLoS genetics 11, e1005612, doi:10.1371/journal.pgen.1005612 (2015).

407

408

Rodrigues, J. V. et al. Biophysical principles predict fitness landscapes of drug resistance. Proceedings of the National Academy of Sciences of the United States of America 113, E14701478, doi:10.1073/pnas.1601441113 (2016).

Gong, L. I., Suchard, M. A. \& Bloom, J. D. Stability-mediated epistasis constrains the evolution of an influenza protein. Elife 2, e00631, doi:10.7554/eLife.00631 (2013). Orr, H. A. Fitness and its role

Elena, S. F. \& Lenski, R. E. Evolution experiments with microorganisms: the dynamics and genetic bases of adaptation. Nature reviews. Genetics 4, 457-469, doi:10.1038/nrg1088 (2003). (1983).

420

421

422

423

424

425

426

427

Biel, S. W. \& Hartl, D. L. Evolution of transposons: natural selection for Tn5 in Escherichia coli K12. Genetics 103, 581-592 (1983).

12 Fridman, O., Goldberg, A., Ronin, I., Shoresh, N. \& Balaban, N. Q. Optimization of lag time underlies antibiotic tolerance in evolved bacterial populations. Nature 513, 418-421, doi:10.1038/nature13469 (2014).

13 Bajaj, K., Chakrabarti, P. \& Varadarajan, R. Mutagenesis-based definitions and probes of residue burial in proteins. Proceedings of the National Academy of Sciences of the United States of America 102, 16221-16226, doi:10.1073/pnas.0505089102 (2005).

428

429 Semisotnov, G. V. et al. Study of the "molten globule" intermediate state in protein folding by a hydrophobic fluorescent probe. Biopolymers 31, 119-128, doi:10.1002/bip.360310111 (1991).

15 Navarro, S. \& Ventura, S. Fluorescent dye ProteoStat to detect and discriminate intracellular amyloid-like aggregates in Escherichia coli. Biotechnol J 9, 1259-1266, doi:10.1002/biot.201400291 (2014). (1987). Bacteria. Cell 165, 1493-1506, doi:10.1016/j.cell.2016.05.003 (2016). 
18 Hegreness, M., Shoresh, N., Hartl, D. \& Kishony, R. An equivalence principle for the incorporation of favorable mutations in asexual populations. Science 311, 1615-1617, doi:10.1126/science.1122469 (2006).

19 Cha, R. S., Zarbl, H., Keohavong, P. \& Thilly, W. G. Mismatch amplification mutation assay (MAMA): application to the C-H-ras gene. PCR methods and applications 2, 14-20 (1992).

20 Jiang, L., Mishra, P., Hietpas, R. T., Zeldovich, K. B. \& Bolon, D. N. Latent effects of Hsp90 mutants revealed at reduced expression levels. PLoS genetics 9, e1003600, doi:10.1371/journal.pgen.1003600 (2013).

21 Serohijos, A. W. \& Shakhnovich, E. I. Merging molecular mechanism and evolution: theory and computation at the interface of biophysics and evolutionary population genetics. Curr Opin Struct Biol 26, 84-91, doi:10.1016/j.sbi.2014.05.005 (2014).

22 Serohijos, A. W., Lee, S. Y. \& Shakhnovich, E. I. Highly abundant proteins favor more stable 3D structures in yeast. Biophys J 104, L1-3, doi:10.1016/j.bpj.2012.11.3838 (2013). Hartl, D. L., Clark, A.G. Principles of population genetics, 4th ed., (Sinauer, 2007). Manhart, M., Adkar, B. V. \& Shakhnovich, E. I. Tradeoffs between microbial growth phases lead to frequency-dependent and non-transitive selection. bioRxiv, doi:10.1101/096453 (2016).

25 Lambert, G., Liao, D., Vyawahare, S. \& Austin, R. H. Anomalous spatial redistribution of competing bacteria under starvation conditions. Journal of bacteriology 193, 1878-1883, doi:10.1128/JB.01430-10 (2011).

26 Palkova, Z. Multicellular microorganisms: laboratory versus nature. EMBO reports 5, 470-476, doi:10.1038/sj.embor.7400145 (2004).

27 Hibbing, M. E., Fuqua, C., Parsek, M. R. \& Peterson, S. B. Bacterial competition: surviving and thriving in the microbial jungle. Nature reviews. Microbiology $\mathbf{8}$, 15-25, doi:10.1038/nrmicro2259 (2010).

28 Todar, K. Textbook of bacteriology. (2015).

29 Adkar, B. V. et al. Protein model discrimination using mutational sensitivity derived from deep sequencing. Structure 20, 371-381, doi:10.1016/j.str.2011.11.021 (2012).

30 Muller, C. W., Schlauderer, G. J., Reinstein, J. \& Schulz, G. E. Adenylate kinase motions during catalysis: an energetic counterweight balancing substrate binding. Structure 4, 147-156 (1996).

\section{Acknowledgements:}

We thank Shimon Bershtein and Adrian Serohijos for helpful discussions.

Contributions:

BVA and EIS - designed research; BVA, SB, JT and MMu - performed experiments; BVA, MMa, SB and EIS - analyzed the data; BVA, MMa, SB and EIS - wrote the paper; All authors edited and approved the final version. 
Fig. 1

A

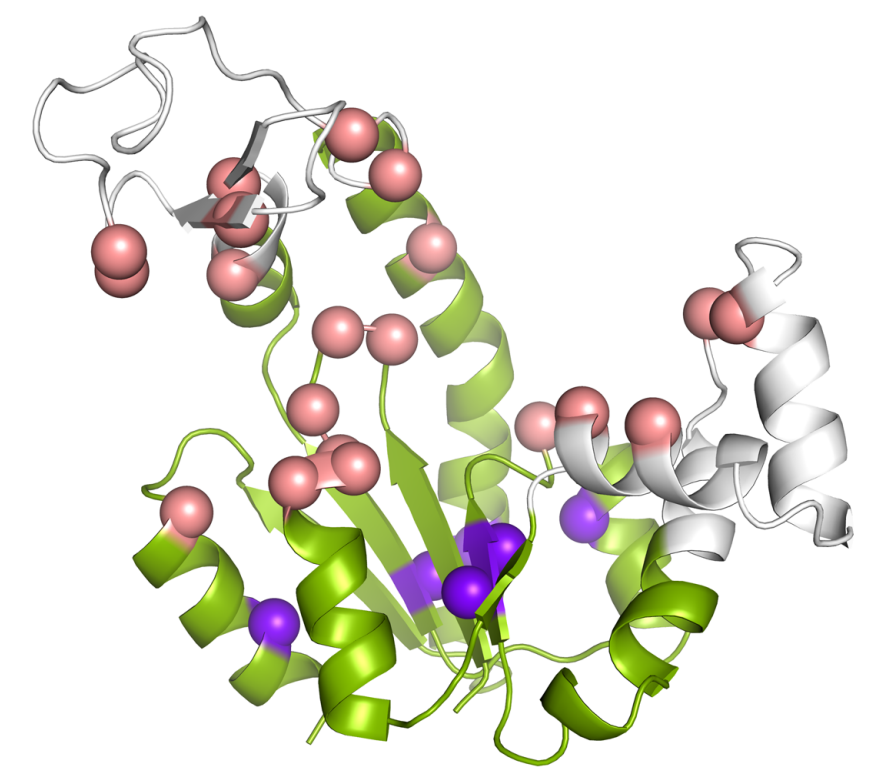

C

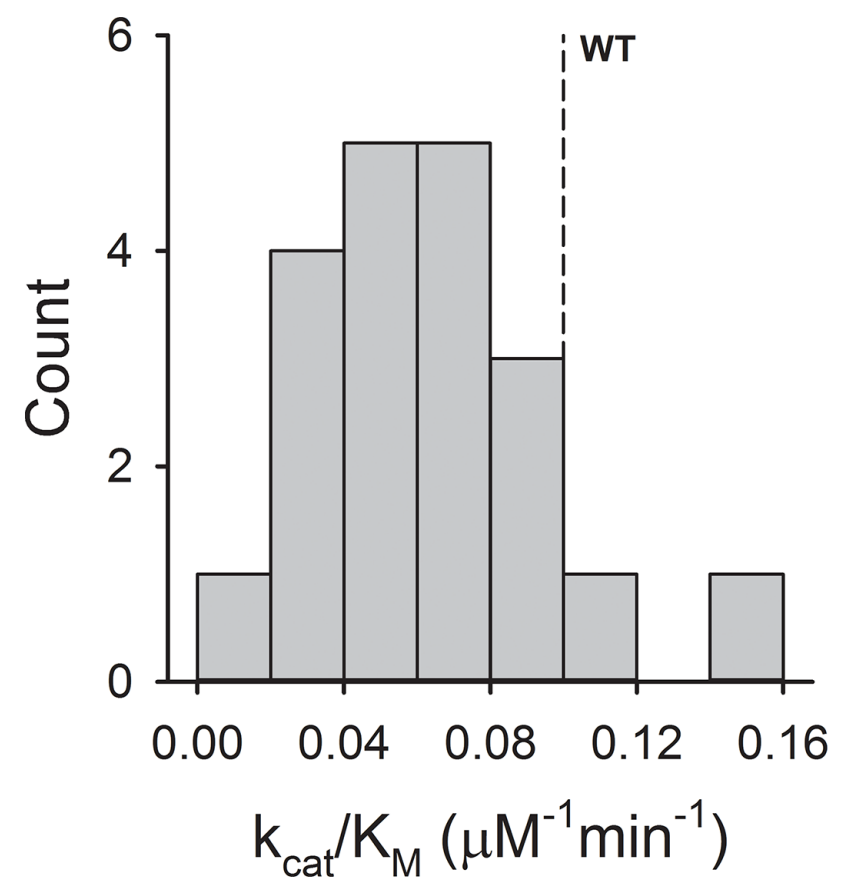

B
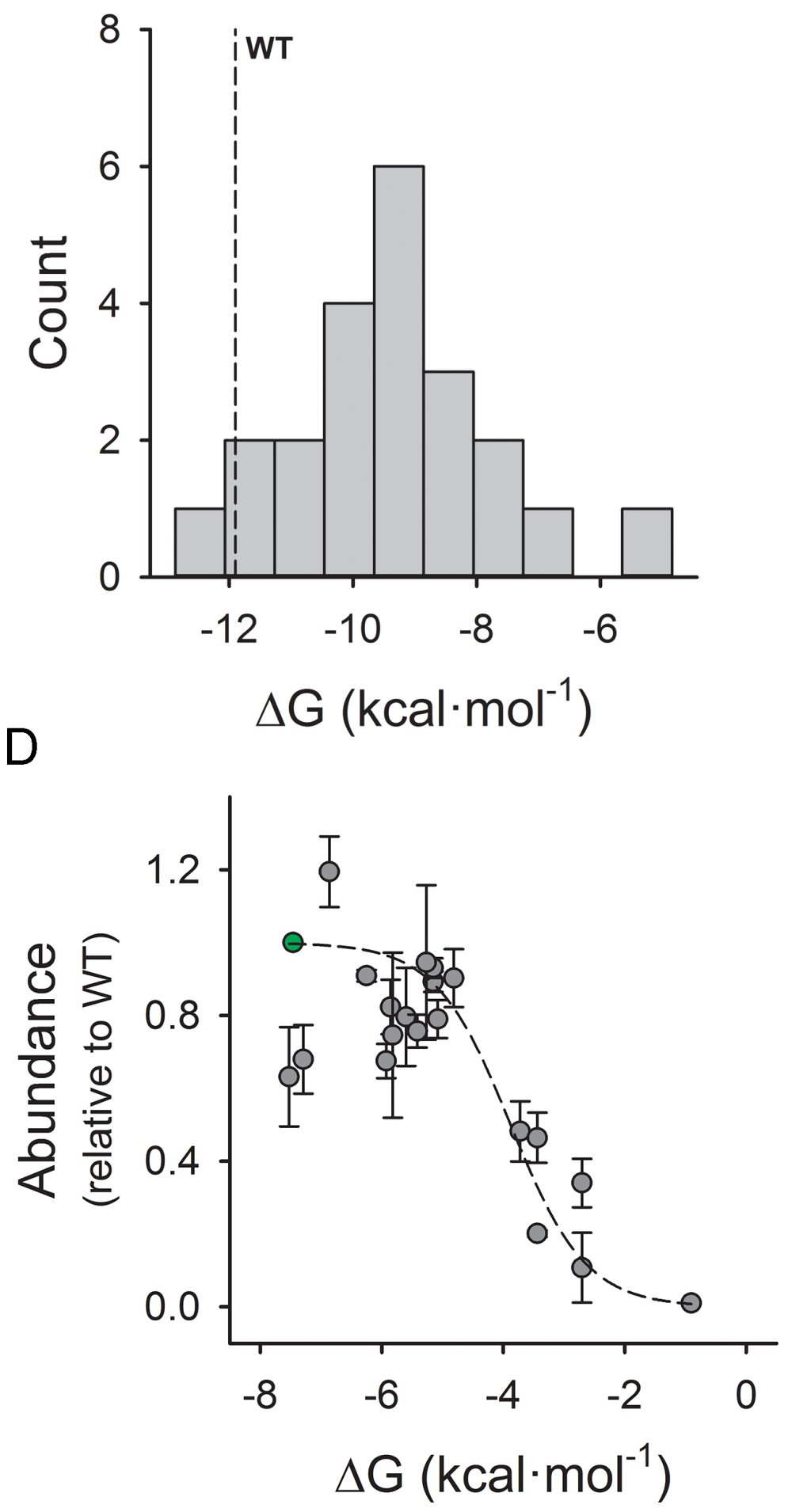
Fig. 2

A

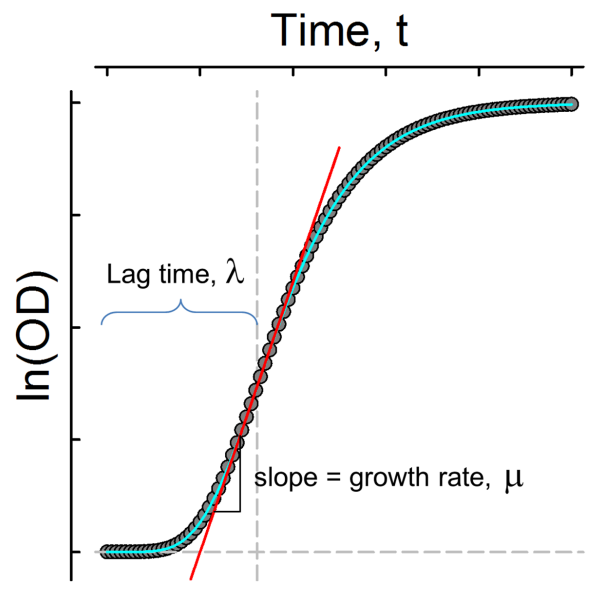

B

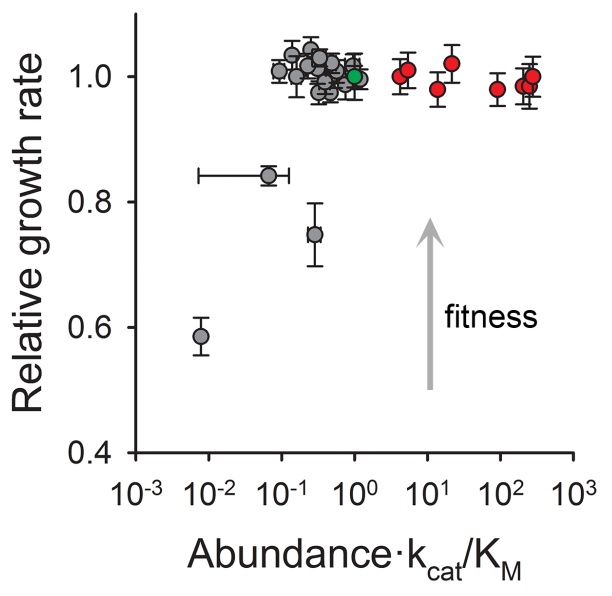

C

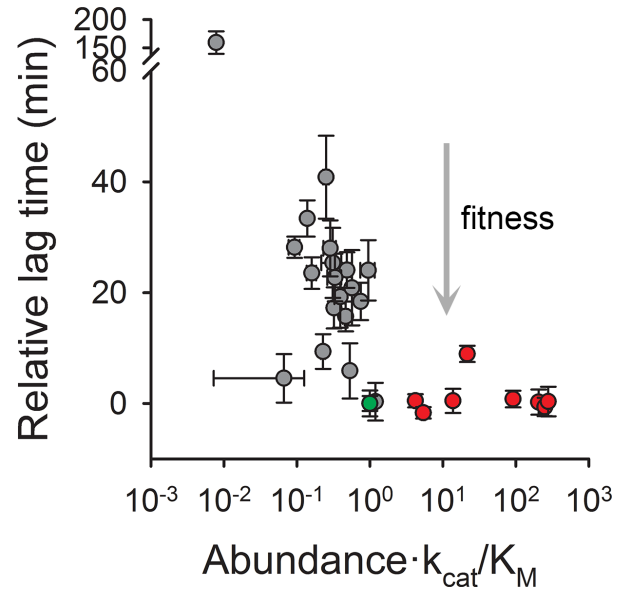


Fig. 3

A

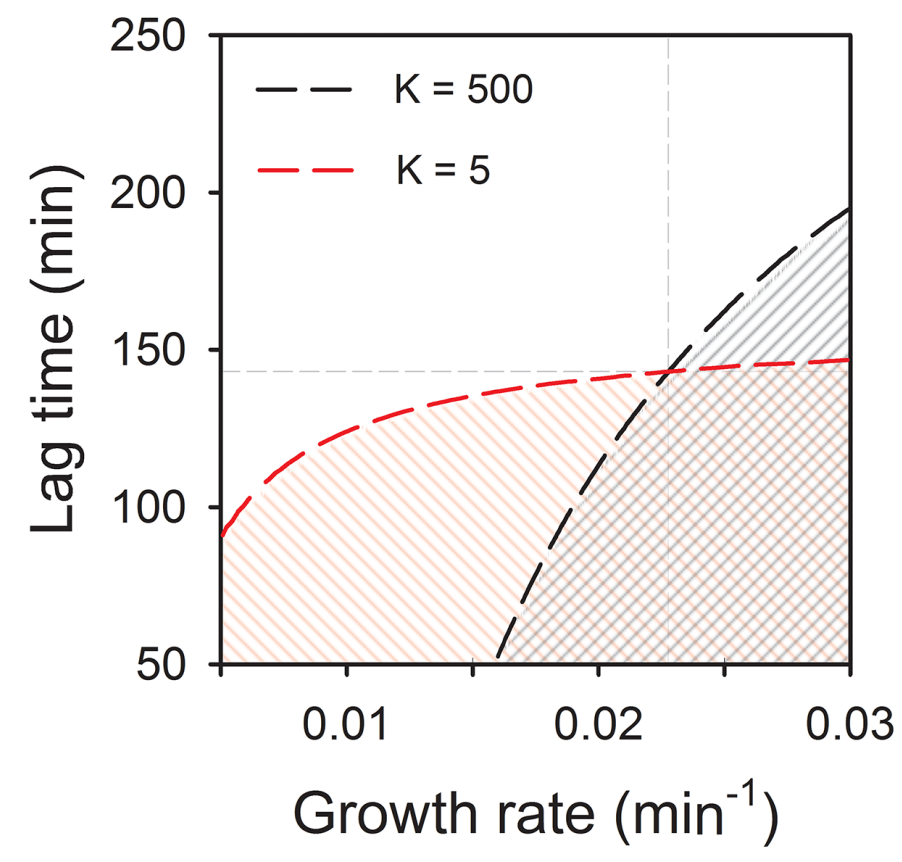

B

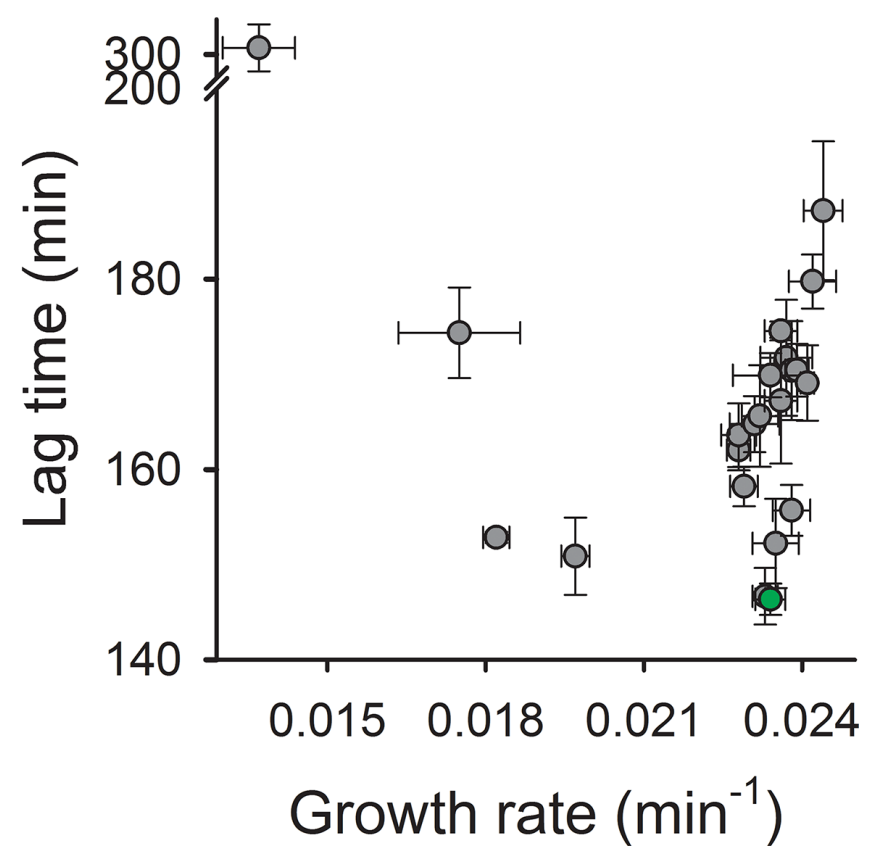


Fig. 4

A

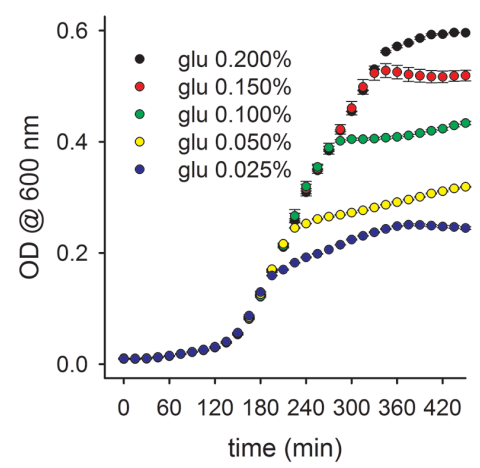

B

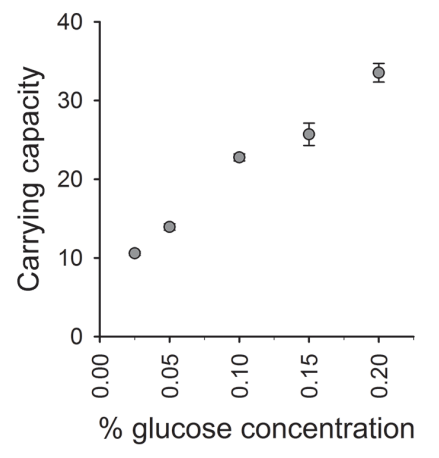

C

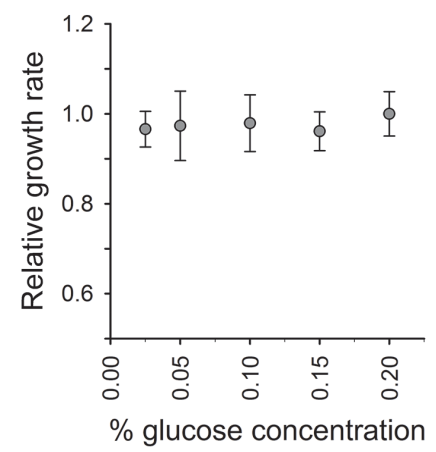

D

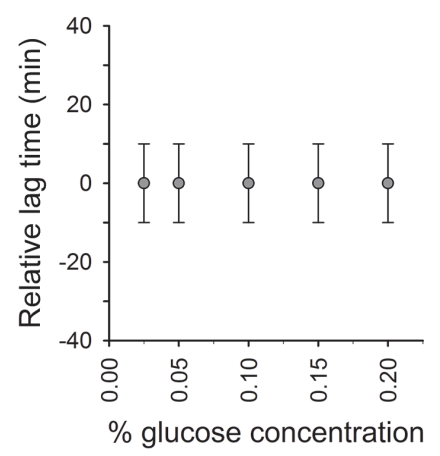


Fig. 5

A

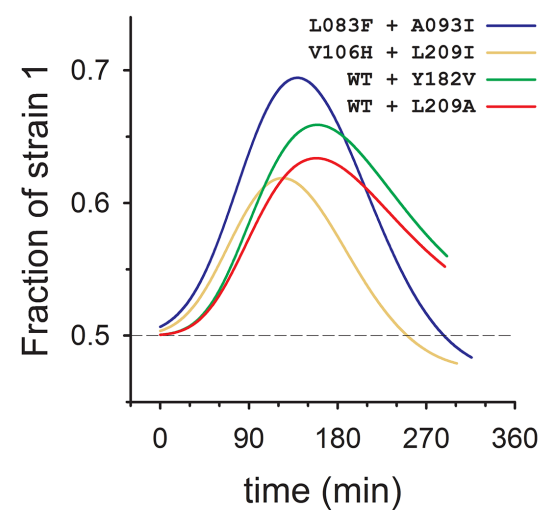

B

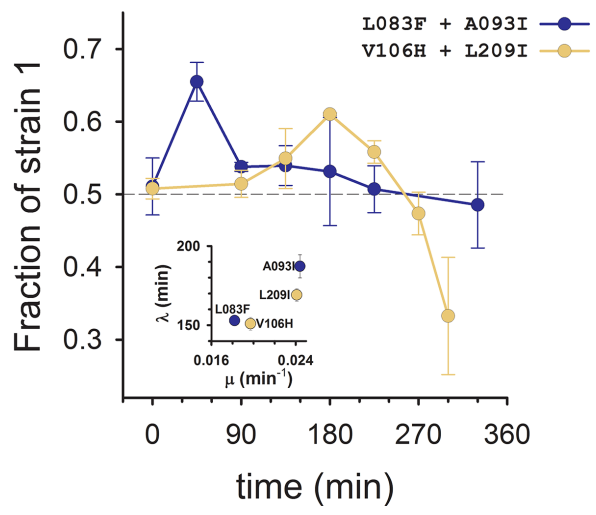

C

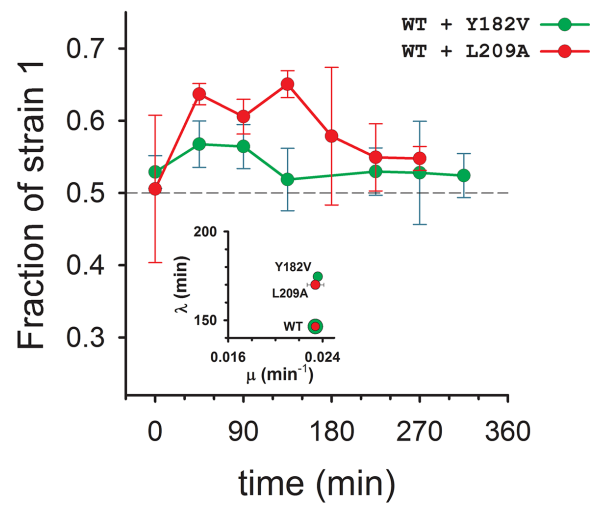




\section{Supplementary Methods}

\section{Mutagenesis and protein purification}

Adenylate kinase (Adk) is encoded by the adk gene, which was cloned under the T7-lac promoter in pET28a $(+)$ vector (Invitrogen) between $N d e \mathrm{I}$ and XhoI restriction sites. We carried out mutagenesis with a pair of 30-35 bp long, partially-complementary primers and the inverse PCR technique using KOD hot-start DNA polymerase. The mutations were centered in the complementary regions of the primers. The mutagenic plasmids were transformed in E.coli DH5 $\alpha$ cells for faithful propagation and storage, and in E. coli BL21(DE3) for protein overexpression and purification. The His-tagged proteins were purified by Ni-NTA affinity chromatography (Qiagen) and subsequently passed through a HiLoad Superdex 75 pg column (GE). The monomeric peak was collected, concentrated and eventually stored in $10 \mathrm{mM}$ potassium phosphate buffer ( $\mathrm{pH}$ 7.2). The concentration of the proteins was measured by BCA assay (ThermoScientific) with BSA as standard.

\section{Biophysical characterization}

Thermal denaturation: We assessed the thermal stability of WT and mutant proteins by differential scanning calorimetry (nanoDSC, TA instruments) using $20 \mu \mathrm{M}$ of protein. The scans were carried out from 10 to $90{ }^{\circ} \mathrm{C}$ at a scan rate of $90{ }^{\circ} \mathrm{C} / \mathrm{hr}$. The thermodynamic parameters were derived by fitting the data to a two-state unfolding model using NanoAnalyze (TA instruments). We also carried out thermal denaturation using the melt-curve module of BioRad CFX96, with Sypro Orange dye as a probe for unfolding as described earlier ${ }^{1}$. The dye was added to the final concentration of $5 \times$ in a $25 \mu 1$ reaction volume containing $4 \mu \mathrm{M}$ of protein in $10 \mathrm{mM}$ potassium phosphate buffer $(\mathrm{pH}$ 7.2). The data were fit to a standard four-parameter sigmoidal equation to obtain apparent melting temperatures.

Urea denaturation: We carried out isothermal urea denaturation with WT and mutant proteins to assess the stability of the proteins to chemical denaturants. We incubated $5 \mu \mathrm{M}$ of protein for $\sim 4 \mathrm{hrs}$ at $25^{\circ} \mathrm{C}$ with varying concentrations of urea $(0-8 \mathrm{M})$. The urea concentrations were estimated by refractive index measurements. The denaturation was monitored by measuring the ellipticity at $222 \mathrm{~nm}$ using a CD spectrometer (Jasco). The melt data was fitted assuming a model 
of two-state unfolding with linear free energy as described earlier ${ }^{2,3}$. The m-value was fixed to $3300 \mathrm{cal} / \mathrm{mol} / \mathrm{M}$ for fitting.

Gel filtration: We assessed the oligomeric status of purified proteins by gel filtration using $50 \mu \mathrm{g}$ of protein on sephadex 75 analytical columns.

ANS and proteostat binding: We used $12 \mu \mathrm{M}$ of bisANS for assessing binding to $2 \mu \mathrm{M}$ of protein in $10 \mathrm{mM}$ potassium phosphate buffer ( $\mathrm{pH}$ 7.2). The excitation and emission wavelengths were set to $395 \mathrm{~nm}$ and $490 \mathrm{~nm}$, respectively. $2 \mu \mathrm{M}$ of protein was incubated with $3.5 \mathrm{mM}$ of the proteostat dye in $1 \times$ assay buffer (Enzo LifeSciences). For this the excitation and emission wavelengths were set to 550 and $600 \mathrm{~nm}$, respectively.

Enzyme activity: We measured the activity of Adk in terms of ADP formation by an end-point assay as described earlier ${ }^{4}$. Briefly, the concentration of AMP was fixed to $500 \mu \mathrm{M}$ and ATP concentration was varied from 0 to $500 \mu \mathrm{M}$ in an enzymatic reaction. $5 \mathrm{nM}$ of Adk was used to initiate the reaction and $500 \mu \mathrm{M}$ of Ap5A was used for quenching at 20, 40, and 60 second time points. The amount of ADP formed was measured by LDH-Pyruvate kinase-coupled reaction and the kinetic parameters were derived by fitting the data to the Michaelis-Menten equation.

Adk overexpression: The $a d k$ gene was cloned in a pBAD plasmid and transformed in the E. coli BW27783 strain (CGSC\#12119). This strain constitutively expresses the arabinose transporter (araE) which enables uniform uptake of arabinose. The cells were induced with increasing concentrations of arabinose from 0 to $0.05 \%$.

Intracellular protein abundance: Cells were grown in supplemented M9 medium for 4 hours at $37^{\circ} \mathrm{C}$, harvested and subsequently lysed with $1 \times$ BugBuster (Novagen) and $25 \mathrm{units} / \mathrm{ml}$ of Benzonase. Total amount of proteins in cell lysate was estimated by BCA assay. The specific fraction of Adk was determined by SDS-PAGE followed by western blot using rabbit anti-Adk polyclonal antibodies (custom- raised by Pacific Immunology).

Estimation of viable cells in saturating culture: The overnight culture was grown in supplemented M9 medium for 16 hours at $30{ }^{\circ} \mathrm{C}$ and the proportion of live:dead cells was measured using Live/Dead BacLight Bacterial Viability Kits (Molecular Probes) according to the manufacturer's instructions. Briefly, $1 \times 10^{8}$ cells (in a volume of $1 \mathrm{ml}$ ) were mixed with $3 \mu 1$ of a 
1:1 proportion of Syto9 dye and Propidium Iodide (PI). The mixture was incubated in the dark for 15 minutes, following which the fluorescence was measured at $530 \mathrm{~nm}$ and $630 \mathrm{~nm}$. Syto9 dye stains live cells and emits fluorescence at $530 \mathrm{~nm}$ (green), while PI stains dead cells and can be detected at $630 \mathrm{~nm}$ (red). The ratio of fluorescence values at $530 \mathrm{~nm}: 630 \mathrm{~nm}$ corresponds to the proportion of live:dead cells in that sample which was eventually used to estimate the percentage of live cells in a sample, according to the manufacturer's instructions. An exponentially growing culture (considered as 100\% live) and cells treated with $70 \%$ ethanol for 1 hour (considered 100\% dead) were mixed in different known proportions, and their 530:630 nm ratio was used to generate a standard curve.

1. Niesen, F.H., Berglund, H. \& Vedadi, M. The use of differential scanning fluorimetry to detect ligand interactions that promote protein stability. Nat Protoc 2, 2212-21 (2007).

2. Chen, B.L. \& Schellman, J.A. Low-temperature unfolding of a mutant of phage T4 lysozyme. 1. Equilibrium studies. Biochemistry 28, 685-91 (1989).

3. Schellman, J.A. The thermodynamic stability of proteins. Annu Rev Biophys Biophys Chem 16, 115-37 (1987).

4. Pena, M.I., Davlieva, M., Bennett, M.R., Olson, J.S. \& Shamoo, Y. Evolutionary fates within a microbial population highlight an essential role for protein folding during natural selection. $\mathrm{Mol}$ Syst Biol 6, 387 (2010). 


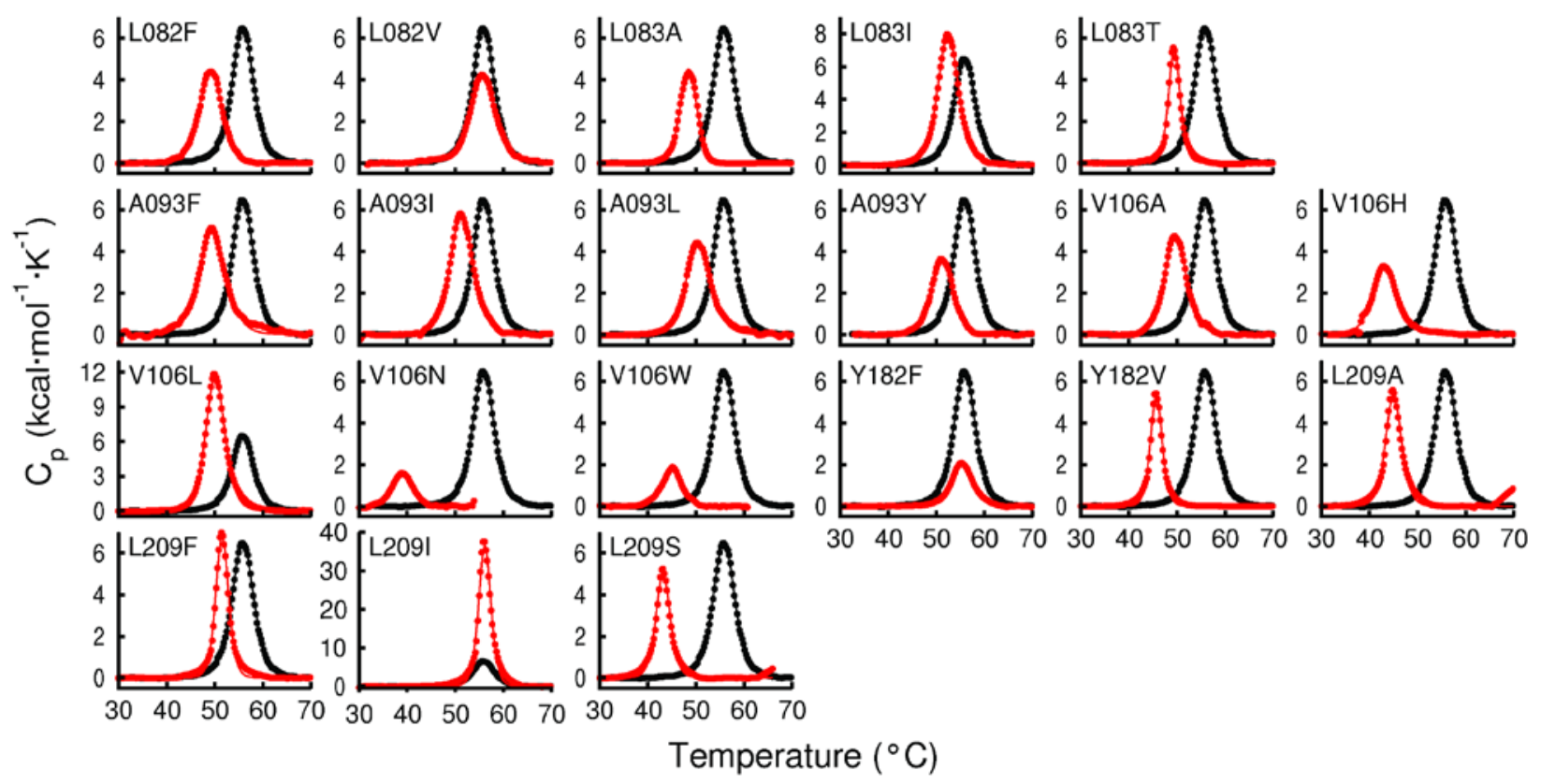

Fig S1: Thermal unfolding monitored by Differential Scanning Calorimetry (DSC) for WT (black trace) and 20 different Adk mutant proteins (red trace). The molar heat capacity $\left(\mathrm{C}_{\mathrm{p}}\right)$ is shown as a function of temperature. The scan rate was $90^{\circ} \mathrm{C} / \mathrm{hr}$. The data was fitted to a twostate thermal unfolding model to derive the thermodynamic parameters. 


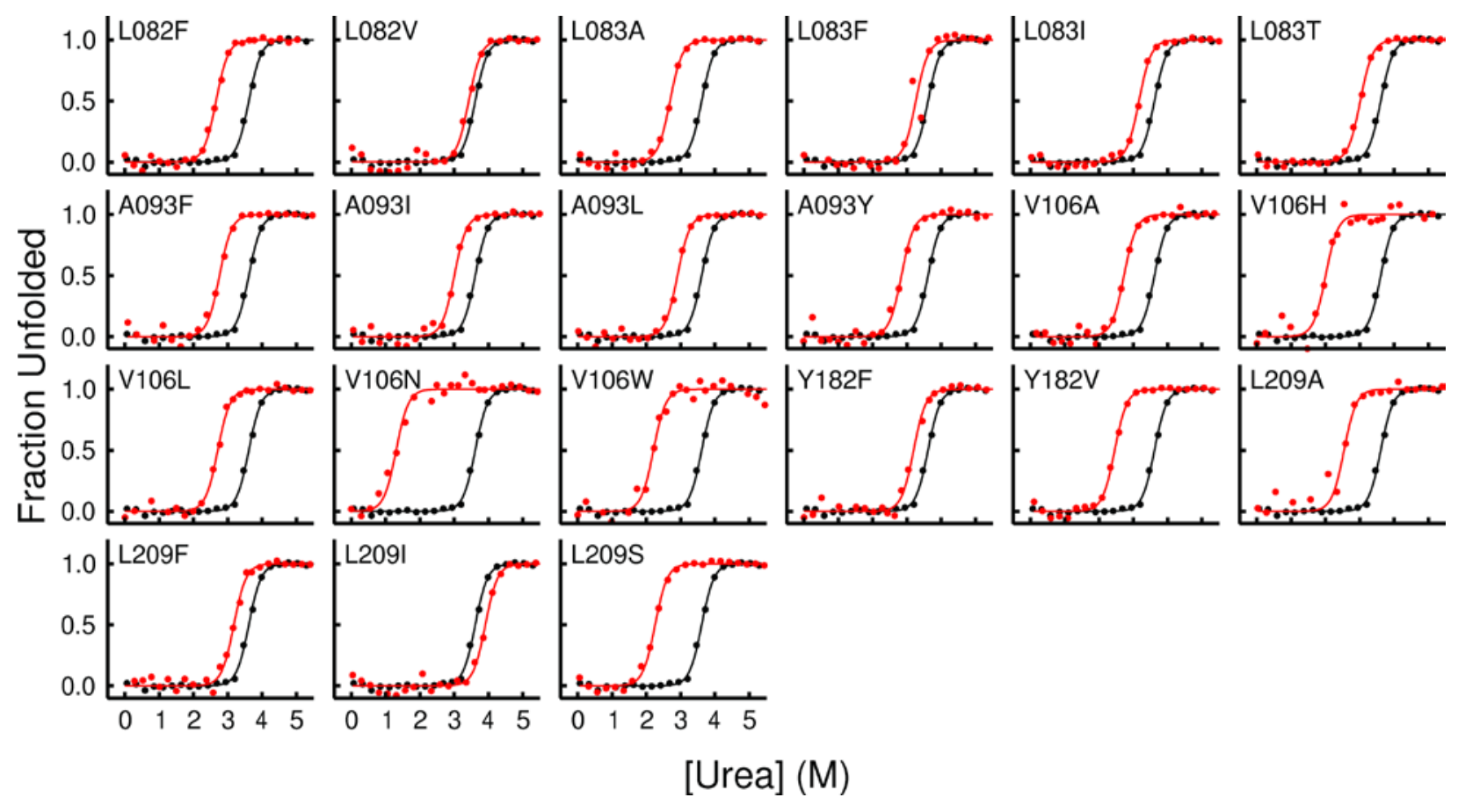

Fig S2: Isothermal urea denaturation curves at $25^{\circ} \mathrm{C}$ for WT (black dots) and mutant Adk proteins (red dots). The fraction unfolded $(\mathrm{Fu})$ is plotted as a function of denaturant concentration. Protein denaturation was monitored by recording the CD signal at $222 \mathrm{~nm}$. The data was fit to a two-state unfolding model. 


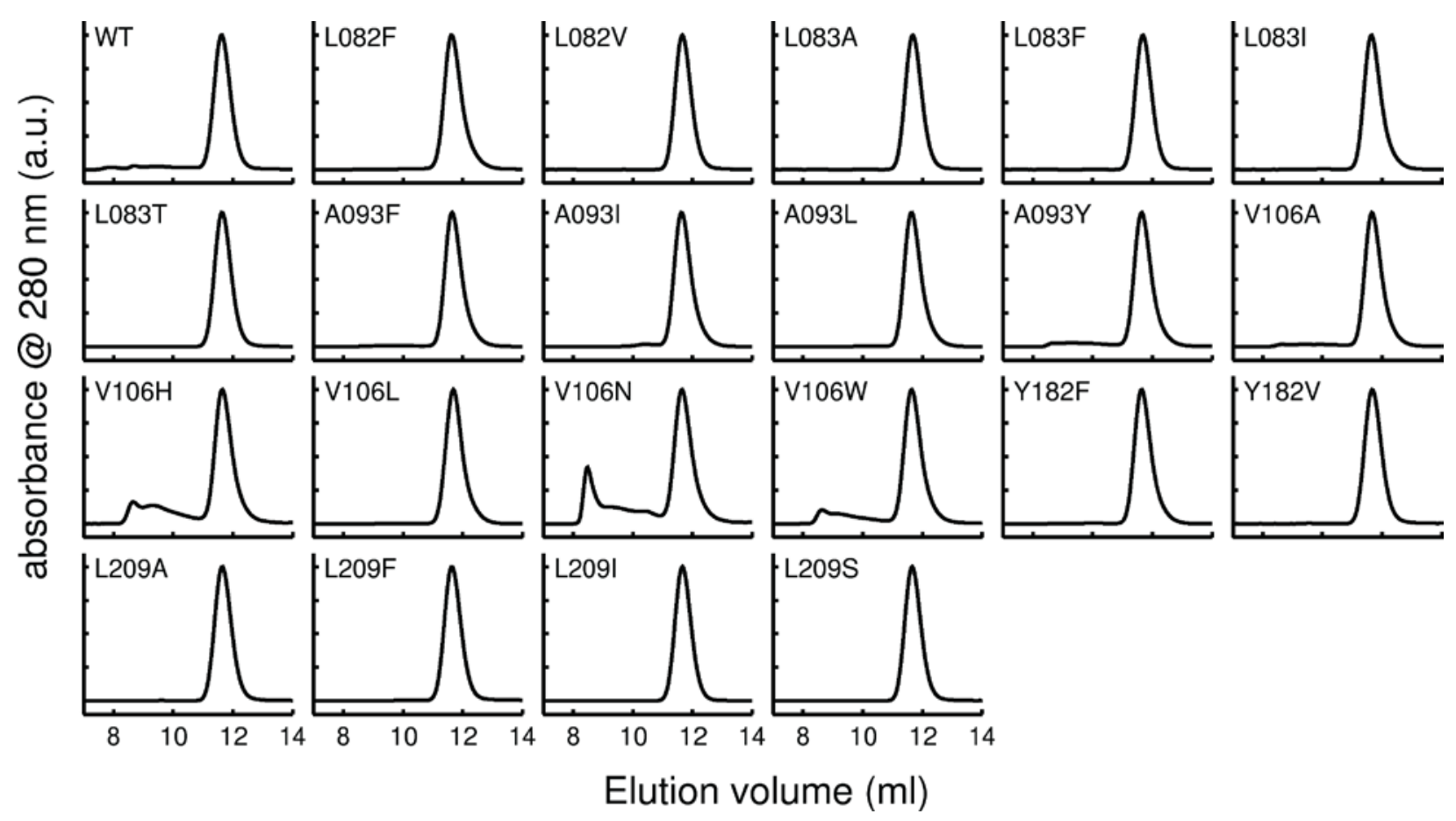

Fig S3: Analytical gel-filtration profile of WT and 20 mutant Adk proteins on a Superdex-75 column at room-temperature. The absorbance at $280 \mathrm{~nm}$ is shown as a function of elution volume. For comparison all the monomeric peaks were normalized to 1. WT Adk along with most other mutant proteins elutes at the expected position for a monomer. Exceptions were V106H, V106N and V106W, where additional peaks appear at much higher molecular weights. 


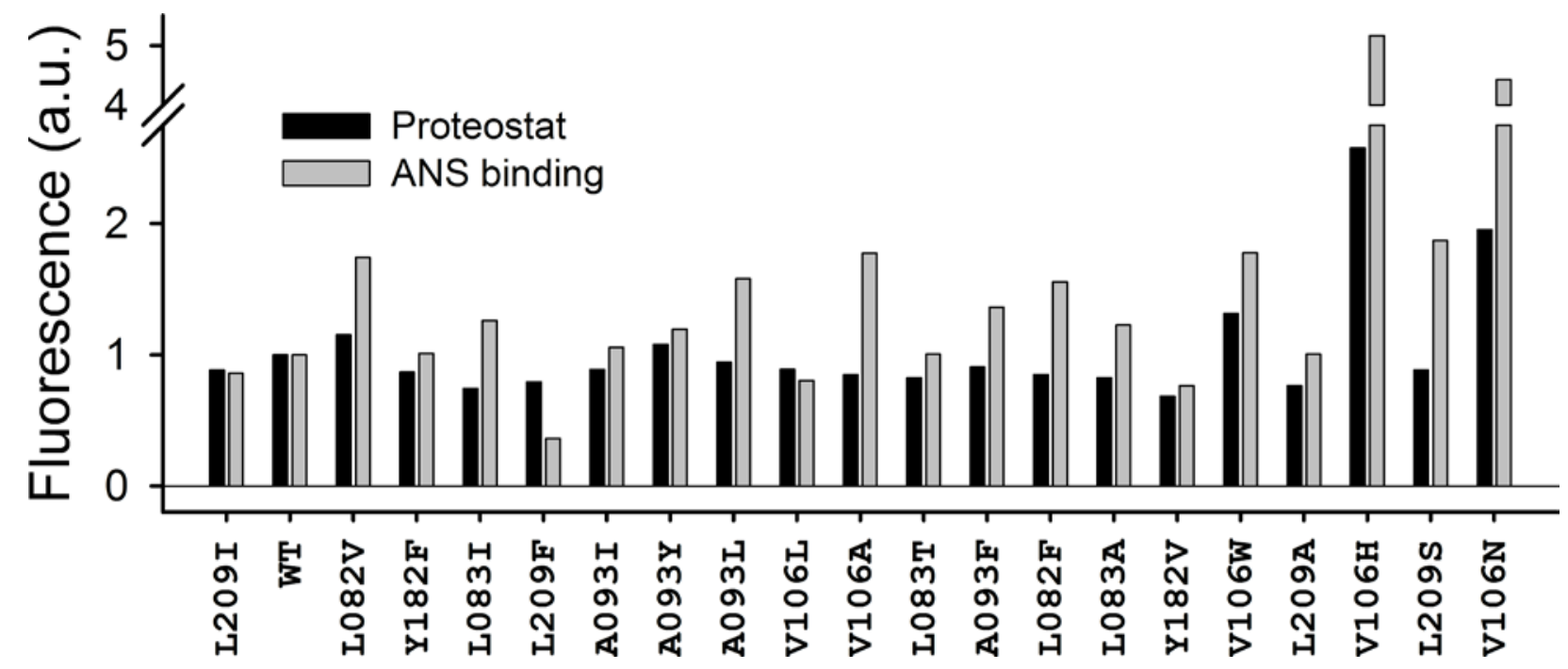

Fig S4: Aggregation propensity and molten-globule states of mutant proteins. Bar plots represent the extent of ProteoStat and ANS binding to WT and mutant Adk proteins. The proteins on the xaxis are arranged in decreasing order of stability from left to right. 


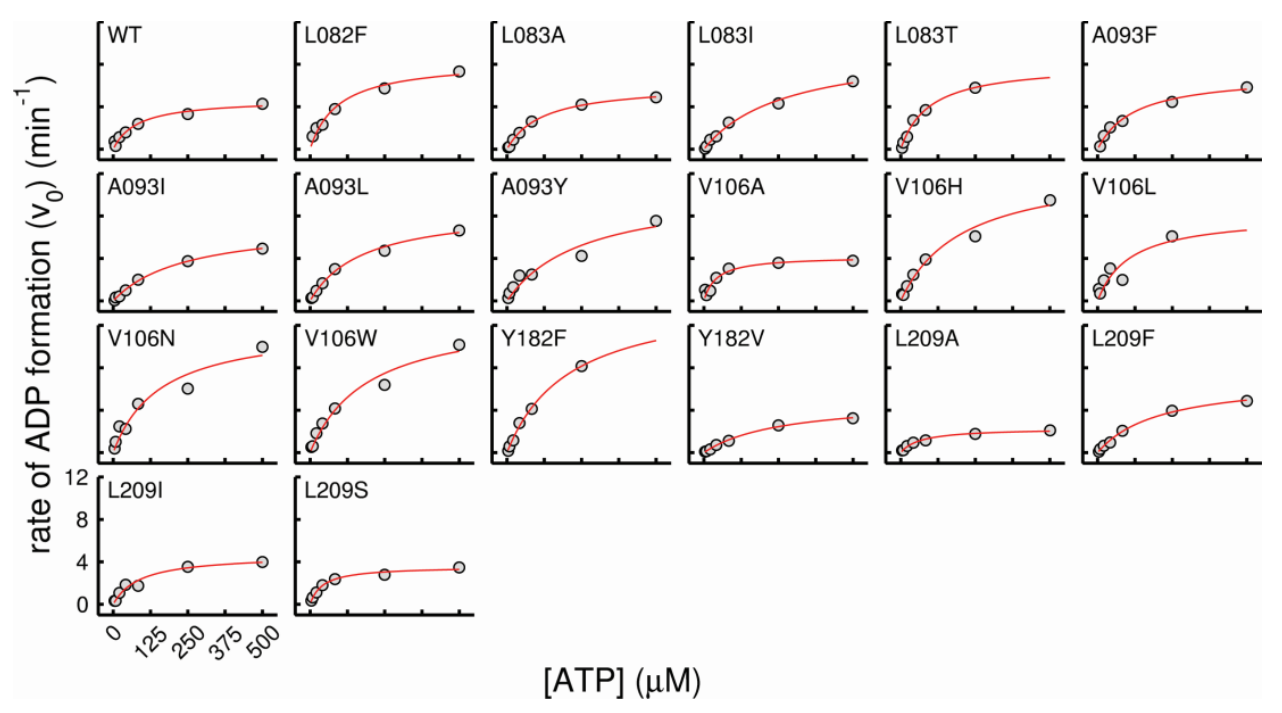

Fig S5: Enzyme activity of Adk mutants at $25^{\circ} \mathrm{C}$ measured as described in Supplementary methods. The initial velocity, shown as a function of ATP concentration, was calculated as the amount of ADP produced per minute by $1 \mathrm{nmol}$ of Adenylate Kinase. The concentration of AMP in all experiments was fixed to $500 \mu \mathrm{M}$. The data (gray circles) was fitted using the MichaelisMenten equation of enzyme activity to extract relevant parameters (fitted line in red). 
A

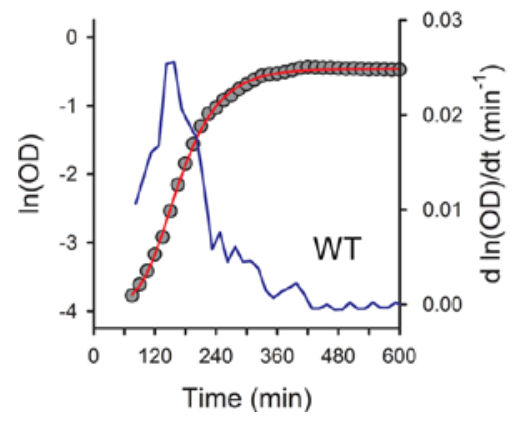

B

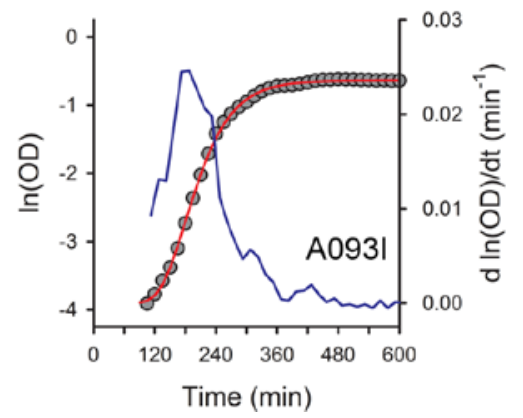

C

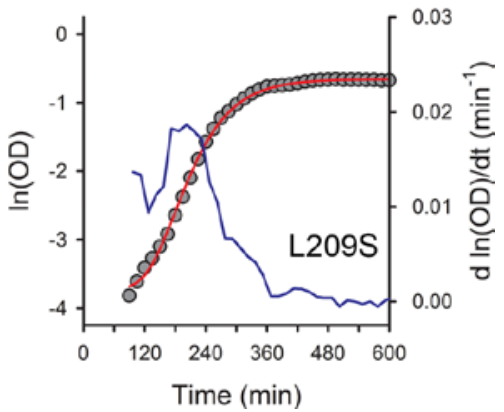

Fig S6: Representative growth curves of (A) WT, (B) A093I, and (C) L209S strains. Each growth curve is shown as $\ln (\mathrm{OD})$ vs time plot (left y-axis). The experimental data is shown in gray circles and the Gompertz fit is shown in solid red line. The instantaneous time derivative of the $\ln (\mathrm{OD})$ data is shown in blue line (right y-axis). The strains were chosen to illustrate the quality of the fit across different range of growth rates and lag times (see Table S2 for growth parameters). 
A

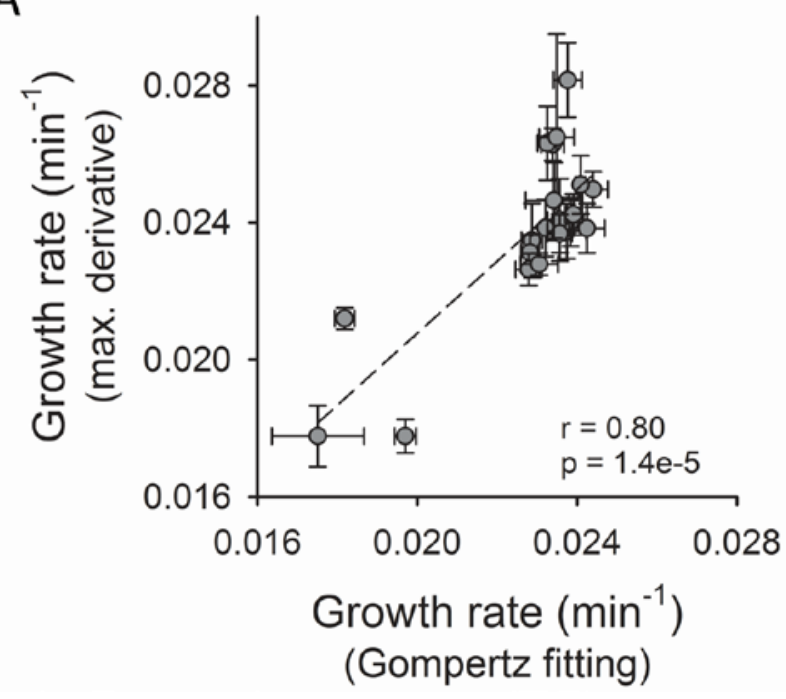

B

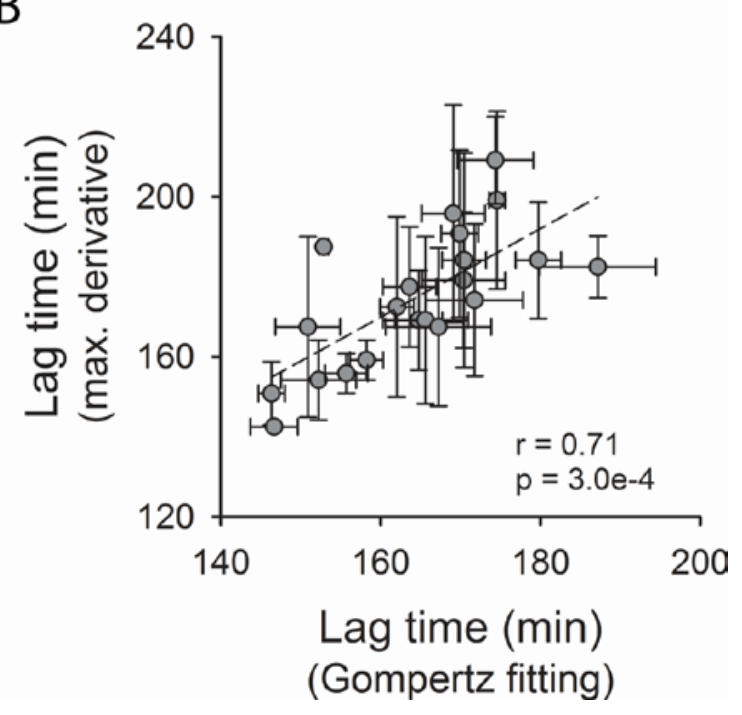

Fig S7: Correlation between growth parameters derived from Gompertz fitting and maximumderivative method. The parameters derived from both the methods correlate very well as indicated by Pearson's correlation parameters ( $\mathrm{r}$ and $\mathrm{p}$-values). The data points represent mean and error bars are standard deviation of 6 or 9 measurements (see Table S2). 
A

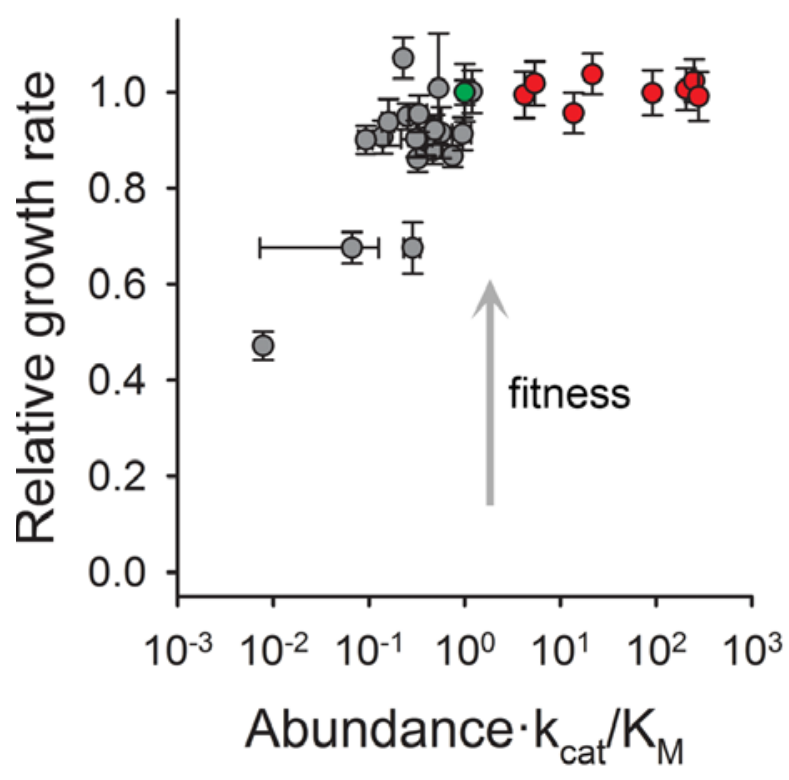

B

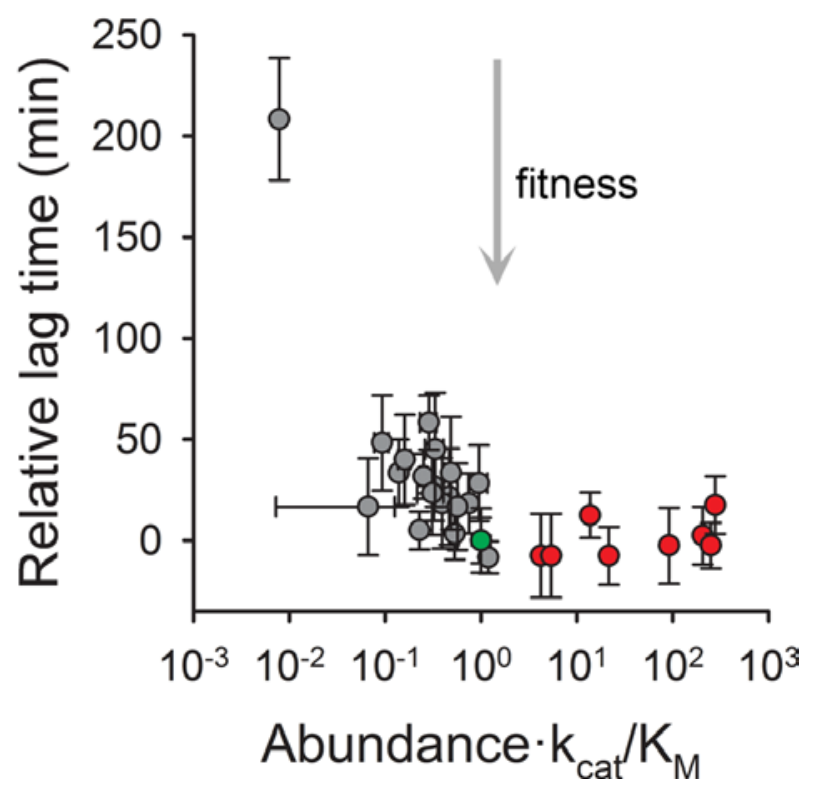

Fig S8: Traits of population growth. (A) Relative growth rates $\left(\mu / \mu_{W T}\right)$ and (B) relative lag time $\left(\lambda-\lambda_{W T}\right)$ obtained from analysis of growth curve derivatives shown as a function of catalytic capacity which is defined as abundance $\times k_{\text {cat }} / K_{M}$. The mutant data is shown in gray circles, whereas red circles represent the BW27783 strain with varying degrees of overexpression of WT Adk from a pBAD plasmid. Data for WT is shown in green. Fig 2 is an equivalent figure with growth rate and lag times obtained after fitting the raw data with Gompertz equation (Eq. 2). 


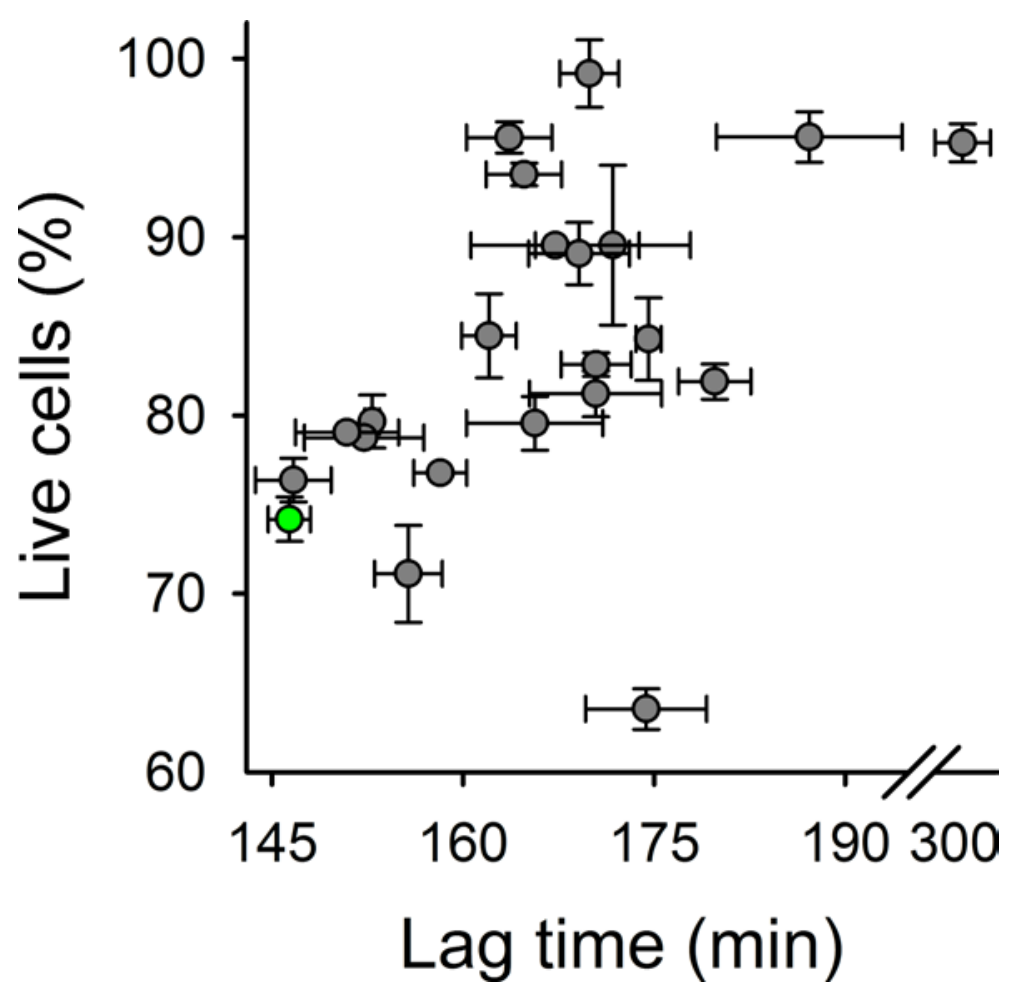

Fig S9: Percentage of live or viable cells of WT and mutant Adk strains at saturation (16 hours of growth) versus their population lag time. The cultures were grown overnight at $30^{\circ} \mathrm{C}$, and then stained using fluorescent dyes Syto9 (specific for live cells) and propidium iodide (specific for dead cells). The data points are mean and error bars represent standard deviation of 2 biological replicates. WT Adk strain is shown in green. 
A
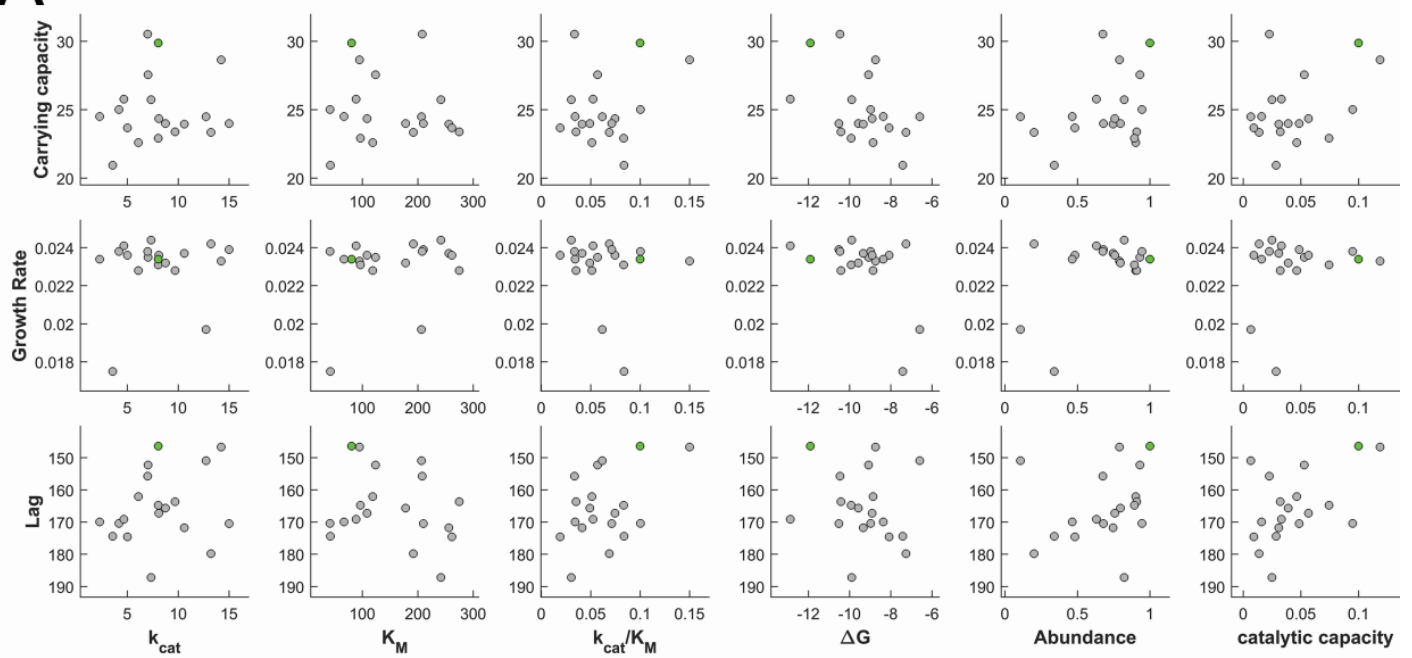

B

\begin{tabular}{cccccccc} 
& & $\begin{array}{c}\mathrm{k}_{\text {cat }} \\
\left(\mathrm{min}^{-1}\right)\end{array}$ & $\begin{array}{c}\mathrm{K}_{\mathrm{M}} \\
(\mu \mathrm{M})\end{array}$ & $\begin{array}{c}\mathrm{k}_{\text {cat }} / \mathrm{K}_{\mathrm{M}} \\
\left(\mu \mathrm{M}^{-1} \cdot \mathrm{min}^{-1}\right)\end{array}$ & $\begin{array}{c}\Delta \mathrm{G} \\
\left(\mathrm{kcal} \cdot \mathrm{mol}^{-1}\right)\end{array}$ & Abundance & $\begin{array}{c}\text { Abundancex } \\
\left(\mathrm{k}_{\text {cat }} / \mathrm{K}_{\mathrm{M}}\right)\end{array}$ \\
\hline Carrying & $\rho$ & -0.02 & -0.17 & 0.04 & -0.38 & 0.22 & 0.24 \\
capacity & $p$-value & 0.943 & 0.495 & 0.870 & 0.108 & 0.375 & 0.314 \\
\hline Growth rate & $\rho$ & 0.04 & 0.20 & -0.21 & -0.32 & -0.07 & -0.08 \\
$\left(\mathrm{~min}^{-1}\right)$ & $p$-value & 0.861 & 0.424 & 0.394 & 0.179 & 0.791 & 0.737 \\
\hline Lag time & $\rho$ & -0.19 & 0.20 & -0.30 & 0.20 & -0.39 & -0.44 \\
$(\mathrm{~min})$ & $p$-value & 0.442 & 0.412 & 0.212 & 0.420 & 0.103 & 0.057 \\
\hline
\end{tabular}

C

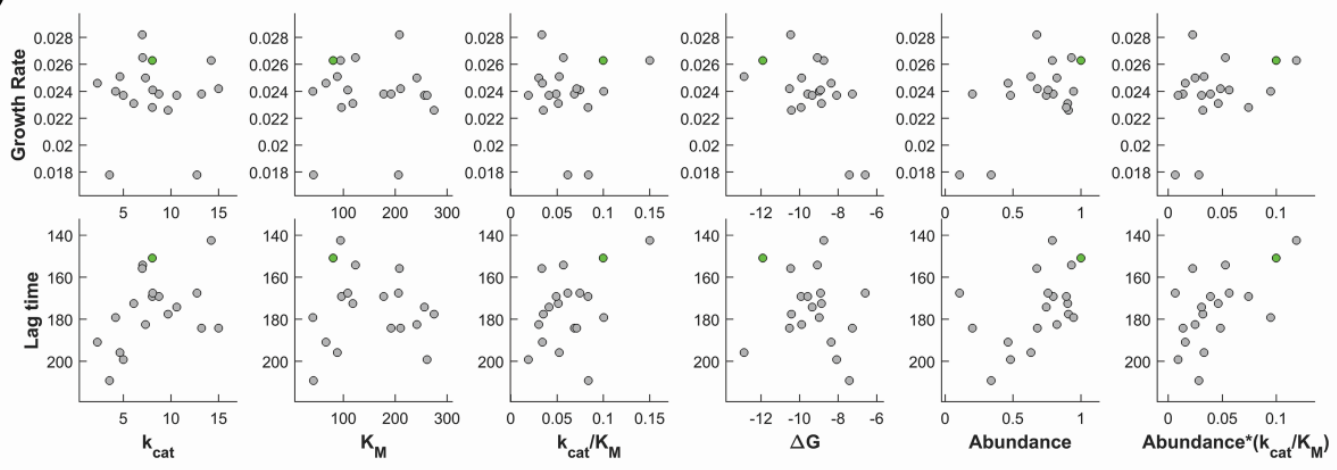

D

\begin{tabular}{cccccccc} 
& & $\begin{array}{c}\mathrm{k}_{\text {cat }} \\
\left(\mathrm{min}^{-1}\right)\end{array}$ & $\begin{array}{c}\mathrm{K}_{\mathrm{M}} \\
(\mu \mathrm{M})\end{array}$ & $\begin{array}{c}\mathrm{k}_{\text {cat }} / \mathrm{K}_{\mathrm{M}} \\
\left(\mu \mathrm{M}^{-1} \cdot \mathrm{min}^{-1}\right)\end{array}$ & $\begin{array}{c}\Delta \mathrm{G} \\
\left(\mathrm{kcal} \cdot \mathrm{mol}^{-1}\right)\end{array}$ & Abundance & $\begin{array}{c}\text { Abundance } \times \\
\left(\mathrm{k}_{\text {cat }} / \mathrm{K}_{\mathrm{M}}\right)\end{array}$ \\
\hline Growth rate & $\rho$ & -0.06 & -0.17 & 0.00 & -0.44 & 0.24 & 0.32 \\
$\left(\mathrm{~min}^{-1}\right)$ & $p$-value & 0.822 & 0.483 & 0.991 & 0.057 & 0.313 & 0.183 \\
\hline Lag time & $\rho$ & -0.42 & 0.03 & -0.30 & 0.17 & -0.48 & -0.51 \\
$(\mathrm{~min})$ & $p$-value & 0.077 & 0.903 & 0.209 & 0.486 & 0.038 & 0.027 \\
\hline
\end{tabular}

Fig S10: Scatter plots of growth parameters (carrying capacity, growth rate and lag times) and molecular and cellular properties of Adk. Parameters were obtained using (A) Gompertz fit and (C) analysis of growth curve derivatives. Panels (B) and (D) show Spearman's correlation coefficients $(\rho)$ and $p$-values for each of the sub-plots in panels (A) and (C) respectively. The 
highest correlation values in each panel are highlighted in yellow. V106N was excluded from all correlation calculations. 
A

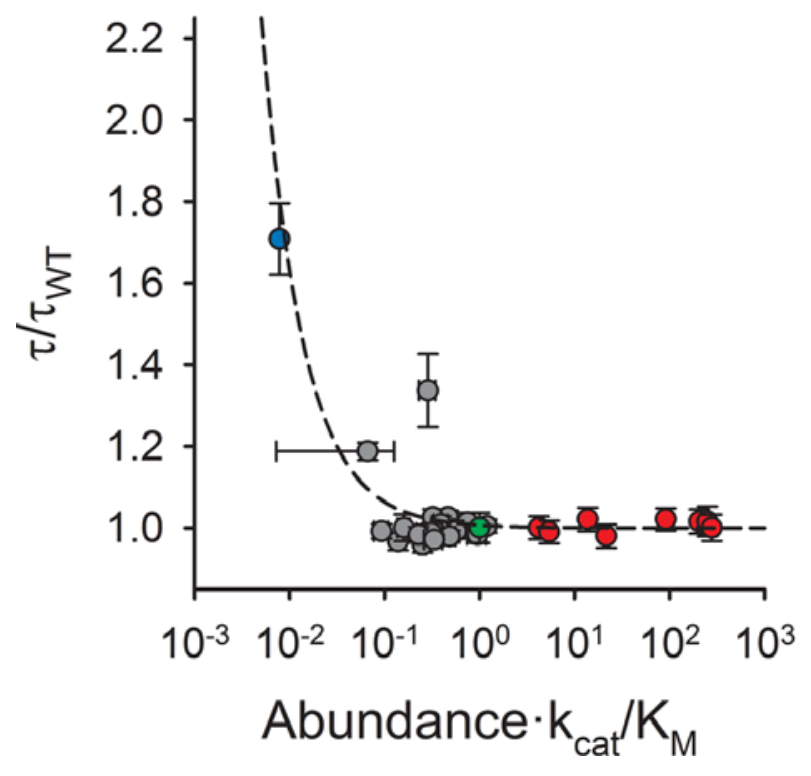

B

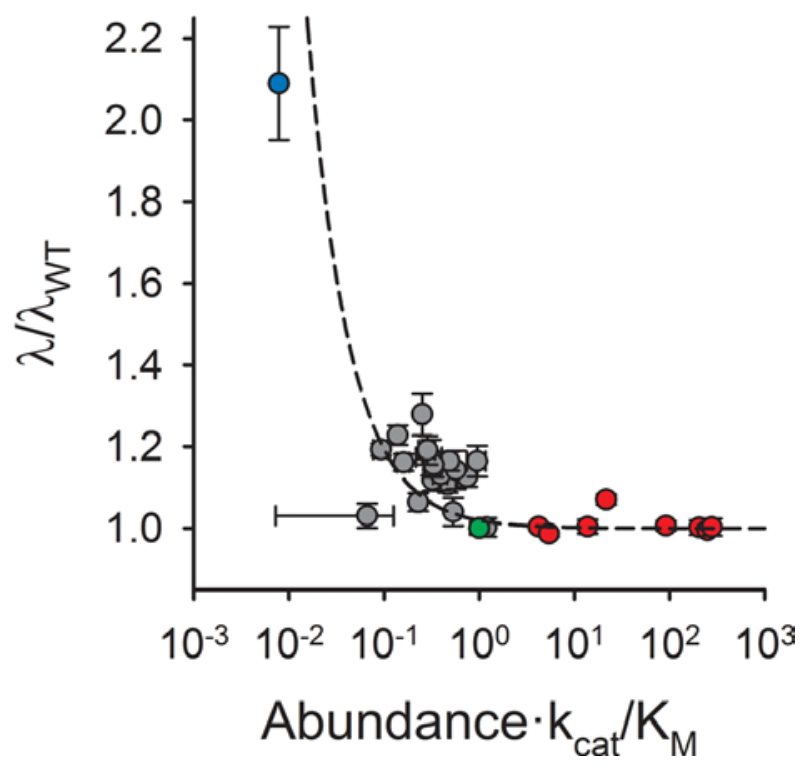

Fig S11: (A) Relative growth time $\left(\tau / \tau_{W T}\right)$ and (B) relative lag time $\left(\lambda / \lambda_{W T}\right)$ as a function of catalytic capacity $\left(\right.$ abundance $\times k_{c a t} / K_{M}$ ). The dashed line shows a fit to Eq. 3 , where the asymptote $(a)$ was assumed to be 1 . The $\mathrm{K}_{\mathrm{M}}$-like parameter $b$ for growth time was 0.006 and that for lag time was 0.019 , which indicates that the WT catalytic capacity is closer to the cusp for lag time than for growth time. The mutant data is shown in gray circles, whereas the overexpression data is shown in red. In green is shown WT, while the blue circle indicates V106N which was omitted from the fitting. The error bars represent standard deviation of parameters derived from growth curves of 3 colonies (biological replicates) in triplicates ( 9 curves). See Table S2 and S3 for the parameters. 


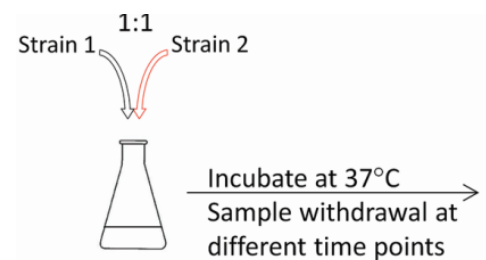

Sample withdrawal at
different time points
Estimation of proportions of individual

strains using qPCR and mismatch

amplification mutation assay (MAMA)

approach

MAMA pcr:

gDNA from strain 1

gDNA from strain 2

$\longrightarrow$ Primer set amplifying only strain 1

Fig S12: Schematic representation of binary growth competition experiments and estimation of relative proportion of competing strains. The strains (1) and (2) are mixed in 1:1 proportion and were grown at $37^{\circ} \mathrm{C}$. Samples were drawn at different time points, normalized for OD, and genomic DNA was extracted. The proportions of individual strains were estimated by a qPCR method employing mismatch amplification mutation assay method (see Methods). We designed a set of primers to differentially amplify the strains by matching the 3'-end of one of the primers to the site of mutation and using Taq DNA polymerase for amplification. 
Table S1: Structural and biophysical parameters

\begin{tabular}{|c|c|c|c|c|c|c|c|c|c|c|}
\hline adk strain & $\begin{array}{r}\mathrm{SC} \operatorname{acc}^{\mathrm{a}} \\
(\%)\end{array}$ & Residue depth (Å) & $\begin{array}{l}\text { Fraction of } \\
\text { WT in MSA }\end{array}$ & $\begin{array}{r}\text { Fraction of } \\
\text { mutant in } \mathrm{MSA}^{\mathrm{b}}\end{array}$ & $\begin{array}{r}T_{m}(D S C) \\
\left({ }^{\circ} \mathrm{C}\right)\end{array}$ & $\begin{array}{r}T_{m}(\text { TFA })^{c} \\
\left({ }^{\circ} \mathrm{C}\right)\end{array}$ & $\begin{array}{r}\Delta \mathrm{G}^{\mathrm{d}} \\
(\mathrm{kcal} / \mathrm{mol})\end{array}$ & $\begin{array}{r}k_{\text {cat }}^{e} \\
\left(\min ^{-1}\right)\end{array}$ & $\begin{array}{c}\mathrm{K}_{\mathrm{M}}^{\mathrm{f}} \\
(\mu \mathrm{M})\end{array}$ & $\begin{array}{r}k_{\text {cat }} / K_{M} \\
\left(\mu M^{-1} \min ^{-1}\right)\end{array}$ \\
\hline WT & -- & -- & -- & -- & 55.9 & 53.8 & -11.9 & 8.05 & 80.58 & $9.99 \mathrm{E}-02$ \\
\hline L082F & 0.0 & 8.8 & 0.57 & 0.00 & 49.2 & 47.4 & -8.7 & 14.23 & 94.86 & $1.50 \mathrm{E}-01$ \\
\hline L082V & 0.0 & 8.8 & 0.57 & 0.14 & 55.7 & 53.2 & -11.3 & n.d. ${ }^{\mathrm{g}}$ & n.d. ${ }^{g}$ & n.d. ${ }^{\mathrm{g}}$ \\
\hline L083A & 0.1 & 9.0 & 0.70 & 0.00 & 48.5 & 46.9 & -8.9 & 6.10 & 118.73 & 5.13E-02 \\
\hline L083F & 0.1 & 9.0 & 0.70 & 0.60 & n.d. ${ }^{\mathrm{g}}$ & 54.2 & -10.8 & n.d..$^{\mathrm{g}}$ & n.d. ${ }^{g}$ & n.d. ${ }^{\mathrm{g}}$ \\
\hline L083। & 0.1 & 9.0 & 0.70 & 0.23 & 52.4 & 50.7 & -10.4 & 9.70 & 274.80 & 3.53E-02 \\
\hline L083T & 0.1 & 9.0 & 0.70 & 0.00 & 49.4 & 49.3 & -9.9 & 8.05 & 96.55 & $8.34 \mathrm{E}-02$ \\
\hline A093F & 0.0 & 5.9 & 0.85 & 0.00 & 49.4 & 47.2 & -9.1 & 7.05 & 123.83 & 5.69E-02 \\
\hline A093I & 0.0 & 5.9 & 0.85 & 0.00 & 51.3 & 49.7 & -9.9 & 7.34 & 241.75 & 3.04E-02 \\
\hline A093L & 0.0 & 5.9 & 0.85 & 0.07 & 50.6 & 47.9 & -9.6 & 8.75 & 178.05 & $4.91 \mathrm{E}-02$ \\
\hline A093Y & 0.0 & 5.9 & 0.85 & 0.00 & 51.2 & 49.4 & -9.3 & 10.61 & 256.23 & 4.14E-02 \\
\hline V106A & 0.0 & 7.4 & 0.74 & 0.38 & 49.7 & 47.3 & -9.0 & 4.19 & 41.78 & $1.00 \mathrm{E}-01$ \\
\hline $\mathrm{V} 106 \mathrm{H}$ & 0.0 & 7.4 & 0.74 & 0.00 & 43.2 & 41.2 & -6.6 & 12.74 & 206.54 & 6.17E-02 \\
\hline V106L & 0.0 & 7.4 & 0.74 & 0.04 & 50.1 & 48.5 & -8.9 & 8.10 & 108.60 & 7.46E-02 \\
\hline V106N & 0.0 & 7.4 & 0.74 & 0.00 & 39.0 & 37.9 & -4.8 & 12.07 & 158.06 & 7.64E-02 \\
\hline V106W & 0.0 & 7.4 & 0.74 & 0.04 & 45.0 & 43.0 & -7.3 & 13.22 & 192.05 & $6.88 \mathrm{E}-02$ \\
\hline Y182F & 7.2 & 6.3 & 0.86 & 0.14 & 55.4 & 53.8 & -10.5 & 15.00 & 210.25 & 7.13E-02 \\
\hline Y182V & 7.2 & 6.3 & 0.86 & 0.00 & 45.7 & 46.2 & -8.1 & 5.01 & 261.53 & $1.92 \mathrm{E}-02$ \\
\hline L209A & 0.0 & 7.0 & 0.23 & 0.08 & 45.0 & 44.3 & -8.4 & 2.30 & 66.94 & 3.43E-02 \\
\hline L209F & 0.0 & 7.0 & 0.23 & 0.01 & 51.5 & 51.0 & -10.5 & 7.00 & 208.17 & 3.36E-02 \\
\hline L2099 ${ }^{h}$ & 0.0 & 7.0 & 0.23 & 0.58 & 56.1 & 55.5 & -12.9 & 4.66 & 88.82 & $5.25 \mathrm{E}-02$ \\
\hline L209S & 0.0 & 7.0 & 0.23 & 0.00 & 43.2 & 42.4 & -7.4 & 3.57 & 42.55 & $8.38 \mathrm{E}-02$ \\
\hline
\end{tabular}

${ }^{a} \%$ sidechain accessibility calculated using coordinates of pdb 4ake

${ }^{b}$ fraction in multiple sequence alignment when WT amino acid is excluded

c melting temperture from thermofluor assay

${ }^{d}$ derived from isothermal urea denaturation experiments at $25 \mathrm{C}$

${ }^{\mathrm{e}}$ kcat for ADP formation

${ }^{\mathrm{f}} \mathrm{KM}$ for ATP

${ }^{\mathrm{g}}$ not determined

${ }^{h}$ the only case in this dataset where fraction of mutant amino acid was greater that WT amino acid in MSA 
Table S2: Intracellular abundance and growth parameters of adk mutants

\begin{tabular}{|c|c|c|c|c|c|c|c|c|}
\hline adk strain & $\begin{array}{r}\text { Carrying } \\
\text { capacity, } \mathrm{K}^{\mathrm{a}}\end{array}$ & s.d. in $K^{b}$ & $\begin{array}{r}\text { Growth rate, } \mu^{\mathrm{a}} \\
\left(\mathrm{min}^{-1}\right)\end{array}$ & s.d. in $\mu^{b}$ & $\begin{array}{r}\text { Lag time, } \lambda^{a, c} \\
(\text { min) }\end{array}$ & s.d. in $\lambda^{b}$ & Abundance $^{d}$ & s.d. in abundance $e^{e}$ \\
\hline WT & 29.9 & 3.0 & 0.0234 & $2.85 \mathrm{E}-04$ & 146.4 & 1.7 & 1.00 & 0.00 \\
\hline L082F & 28.7 & 2.9 & 0.0233 & $2.40 \mathrm{E}-04$ & 146.7 & 3.0 & 0.79 & 0.05 \\
\hline L082V & 24.4 & 0.8 & 0.0229 & $2.60 \mathrm{E}-04$ & 158.2 & 2.1 & n.d. ${ }^{f}$ & n.d. ${ }^{f}$ \\
\hline L083A & 22.6 & 0.8 & 0.0228 & $2.22 \mathrm{E}-04$ & 162.0 & 2.1 & 0.90 & 0.08 \\
\hline $\mathrm{LO} 83 \mathrm{~F}^{\mathrm{g}}$ & 27.3 & 1.3 & 0.0182 & $2.48 \mathrm{E}-04$ & 152.9 & 0.6 & 1.19 & 0.10 \\
\hline L083I & 23.4 & 0.9 & 0.0228 & $3.30 \mathrm{E}-04$ & 163.6 & 3.3 & 0.91 & 0.02 \\
\hline L083T & 22.9 & 1.0 & 0.0231 & $4.72 \mathrm{E}-04$ & 164.8 & 2.9 & 0.89 & 0.03 \\
\hline A093F & 27.6 & 4.3 & 0.0235 & 4.37E-04 & 152.2 & 4.7 & 0.93 & 0.03 \\
\hline$A 0931^{g}$ & 25.7 & 0.5 & 0.0244 & $3.69 \mathrm{E}-04$ & 187.2 & 7.3 & 0.82 & 0.07 \\
\hline A093L & 24.0 & 1.0 & 0.0232 & $3.44 \mathrm{E}-04$ & 165.6 & 5.3 & 0.80 & 0.13 \\
\hline A093Y & 24.0 & 1.1 & 0.0237 & $4.90 \mathrm{E}-04$ & 171.7 & 6.1 & 0.75 & 0.23 \\
\hline V106A & 25.0 & 0.5 & 0.0238 & $3.28 \mathrm{E}-04$ & 170.4 & 5.2 & 0.95 & 0.21 \\
\hline $\mathrm{V} 106 \mathrm{H}^{\mathrm{g}}$ & 24.5 & 2.2 & 0.0197 & $2.68 \mathrm{E}-04$ & 150.9 & 4.1 & 0.11 & 0.10 \\
\hline V106L & 24.4 & 2.2 & 0.0236 & $3.08 \mathrm{E}-04$ & 167.2 & 6.6 & 0.76 & 0.05 \\
\hline V106N & 5.9 & 0.3 & 0.0137 & $6.85 \mathrm{E}-04$ & 305.8 & 20.0 & 0.01 & 0.00 \\
\hline V106W & 23.4 & 1.0 & 0.0242 & $4.48 \mathrm{E}-04$ & 179.7 & 2.8 & 0.20 & 0.01 \\
\hline Y182F & 24.0 & 1.3 & 0.0239 & $2.15 \mathrm{E}-04$ & 170.4 & 2.8 & 0.68 & 0.09 \\
\hline Y182V & 23.7 & 1.1 & 0.0236 & $3.08 \mathrm{E}-04$ & 174.6 & 1.0 & 0.48 & 0.08 \\
\hline L209A & 24.5 & 1.3 & 0.0234 & $7.12 \mathrm{E}-04$ & 169.9 & 2.3 & 0.46 & 0.07 \\
\hline L209F & 30.5 & 1.8 & 0.0238 & $3.54 \mathrm{E}-04$ & 155.7 & 2.7 & 0.68 & 0.05 \\
\hline L209I & 25.8 & 1.2 & 0.0241 & $1.27 \mathrm{E}-04$ & 169.1 & 4.0 & 0.63 & 0.14 \\
\hline L209S & 20.9 & 1.5 & 0.0175 & $1.15 \mathrm{E}-03$ & 174.4 & 4.8 & 0.34 & 0.07 \\
\hline
\end{tabular}

a parameters derived by fitting Gompertz equation to $\ln (\mathrm{OD})$ vs time at $37 \mathrm{C}$

${ }^{\mathrm{b}}$ standard deviation derived from 9 replicates (triplicates of 3 biological replicates)

${ }^{c}$ time required to achieve maximum growth rate

d abundance measured after $4 \mathrm{~h}$ of growth at $37 \mathrm{C}$

e standard deviation derived 2 biological replicates

${ }^{f}$ not determined

${ }^{g}$ growth parameters derived for 6 replicates (triplicates of 2 biological replicates) 
Table S3: Intracellular abundance and growth parameters of WT adk overexpression from pBAD plasmid in E. coli BW27783 strain

\begin{tabular}{|c|c|c|c|c|c|c|c|c|}
\hline $\begin{array}{r}\text { arabinose } \\
\text { concentration } \\
(\%)\end{array}$ & $\begin{array}{r}\text { Carrying capacity, } \\
\qquad \mathrm{K}^{\mathrm{a}}\end{array}$ & s.d. in $K^{b}$ & $\begin{array}{r}\text { Growth rate, } \mu^{a} \\
\left(\min ^{-1}\right)\end{array}$ & s.d. in $\mu^{b}$ & $\begin{array}{r}\text { Lag time, } \lambda^{a, c} \\
(\text { min) }\end{array}$ & s.d. in $\lambda^{b}$ & Abundance $^{d}$ & s.d. in abundance ${ }^{e}$ \\
\hline no plasmid & 29.5 & 2.5 & 0.0195 & $5.10 \mathrm{E}-04$ & 126.7 & 1.0 & 1.00 & 0.10 \\
\hline $0.00 \mathrm{E}+00$ & 32.0 & 0.5 & 0.0195 & $2.00 \mathrm{E}-04$ & 127.2 & 0.7 & 4.20 & 0.42 \\
\hline $3.05 \mathrm{E}-06$ & 30.9 & 1.8 & 0.0191 & $2.00 \mathrm{E}-04$ & 127.2 & 2.0 & 13.73 & 1.37 \\
\hline $1.22 \mathrm{E}-05$ & 31.3 & 1.0 & 0.0197 & $2.00 \mathrm{E}-04$ & 125.0 & 0.4 & 5.39 & 0.54 \\
\hline $4.88 \mathrm{E}-05$ & 27.1 & 1.0 & 0.0199 & $2.65 \mathrm{E}-04$ & 135.7 & 1.1 & 21.55 & 2.15 \\
\hline $1.95 \mathrm{E}-04$ & 32.7 & 0.9 & 0.0191 & $1.00 \mathrm{E}-04$ & 127.5 & 1.2 & 91.27 & 9.12 \\
\hline $7.81 E-04$ & 31.6 & 2.5 & 0.0192 & $2.52 \mathrm{E}-04$ & 127.0 & 2.1 & 205.31 & 20.51 \\
\hline $3.13 E-03$ & 32.6 & 1.6 & 0.0192 & $4.73 E-04$ & 126.1 & 1.4 & 250.39 & 25.02 \\
\hline $5.00 \mathrm{E}-02$ & 28.4 & 3.1 & 0.0195 & $3.61 \mathrm{E}-04$ & 127.1 & 2.5 & 278.69 & 27.84 \\
\hline
\end{tabular}

${ }^{a}$ parameters derived by fitting Gompertz equation to $\ln (\mathrm{OD})$ vs time at $37 \mathrm{C}$

${ }^{\mathrm{b}}$ standard deviation of 3 replicates

${ }^{\mathrm{c}}$ time required to achieve maximum growth rate

$\mathrm{d}$ abundance measured after $4 \mathrm{~h}$ of growth at $37 \mathrm{C}$

e standard deviation of 2 biological replicates 